\title{
Die Entwickelung des häutigen Labyrinthes des Knochenfisches (Oryzias latipes).
}

\author{
Von \\ Terukuni Murayama. \\ Aus dem Anatomischen Institut der Keio Universität, Tokyo.
}

Mit 14 Textfiguren und 26 Figuren auf Tafeln XXXVIII-XL.

\section{Einleitung.}

Die morphologischen und histologischen Verhältnisse des Gehörorgans von Teleostiern wurden seit Aelian us und Plinius von vielen Autoren vielfach untersucht. Dennoch sind die Ansichten nach manchen Seiten hin noch nicht vollkommen übereinstimmend. Besonders bezüglich dessen Entwickelungsgeschichte haben wir relativ wenige Arbeiten, nur die von Vogt ('42), Noorden ('83) und Okajima ('11) sind zu nennen, und Ergänzungen zu diesen sind durchaus erwünscht. So habe ich die vorliegende Untersuchung an Oryzias latipes, einer Art der japanischen Knochenfische, angestellt und dabej meine besondere Aufmerksamkeit auf die Gestaltveränderungen der wichtigen Teile des häutigen Labyrinthes während des Entwickelungsverlaufes gelenkt.

\section{Material und Methode.}

Ich untersuchte die verschiedenen Stadien von Eiern des Oryzias latipes, von 48 Stunden nach der Befruchtung bis zur $18 \mathrm{~mm}$ langen Larve, und die ausgewachsenen Exemplare. Die Eier wurden morgens den 5. August 1925 befruchtet, kurz danach in die Glaskufe gebracht und bis zur Ausbrütung aufgezogen. Die Tiere schlüpften erst 168 Stunden nach der Befruchtung aus den Eischalen. Die Larven wurden mit Formalin oder Formolalkohol fixiert. Als Fixiermittel der ausge'wachsenen Exemplare wurde,Formalin angewandt. Ich habe die Larven alle mit Paraffin eingebettet und grösstenteils in senkrechte Serien geschnitten, auch wurde eine Anzahl von horizontalen Serien angefertigt; die Dicke der Schnitte beträgt 10, 15 oder $20 \mu$. Die ausgewachsenen Exemplare wurden, nachdem sie mit 3\%iger Salpetersäure entkalkt waren, alle in Zelloidin eingebettet und in senkrechte sowie horizontale Serienschnitte von $30 \mu$ Dicke geschnitten. Die Larven wurden mit alkoholi- 
schem Boraxkarmin, Eisenhämatoxylin nach Weigert (Stückfärbung) und die ausgewachsenen Exemplare mit Hämatoxylin-Eosin gefärbt.

Die folgenden Beschreibungen der einzelnen Stadien beruhen auf der Beobachtung von Wachsplattenmodellen und Serienschnitten; ich habe 15 Schnittserien und 12 Modelle des häutigen Labyrinthes von 12 Stadien angefertigt. Dieselben sind entweder 33 oder $100 \mathrm{mal}$ vergrössert modelliert. Die Richtzeichen wurden mittelst des Beschneiderritzers von O kajima ('22) angebracht.

\section{Stadienbeschreibung.}

Ich habe die folgenden Stadien verfolgt.

Stadium 1. 72 Stunden nach Befruchtung.

Stadium 2. 84 Stunden nach Befruchtung.

Stadium 3. 96 Stunden nach Befruchtung.

Stadium 4. 120 Stunden nach Befruchtung.

Stadium 5. 144 Stunden nach Befruchtung, Körperlänge $3 \mathrm{~mm}$.

Stadium 6. 168 Stunden nach Befruchtung, Körperlänge $4 \mathrm{~mm}$.

Stadium 7. 8 Tage nach Ausbrütung, Körperlänge $7 \mathrm{~mm}$.

Stadium 8. 15 Tage nach Ausbrütung, Körperlänge $9 \mathrm{~mm}$.

Stadium 9. 35 Tage nach Ausbrütung, Körperlänge $13 \mathrm{~mm}$.

Stadium 10. Ausgewachsene Exemplare, Körperlänge $34 \mathrm{~mm}$.

Stadium 1. (72 Stunden nach Befruchtung.)

Das Riechfeld zeigt noch keine Andeutung. Das Augenbläschen schliesst schon ein sehr enges Lumen ein, die Linsenplatte ist ausgebildet, aber nicht stark verdickt und die Retina ist etwas pigmentiert.

Das schon geschlossene Hörbläschen (Tafelfig. 1, 2 und Textfig. 1) ist seitlich stark abgeplattet und enthält einen sehr engen Binnenraum. Sein vorderer, oberer Teil deviiert etwas nach aussen.

Auf Schnitten sieht der Binnenraum schmal linienartig aus und ist am vorderen Teil etwas erweitert. Das Hörbläschen steht in sagittaler Richtung und legt sich dem Hirnbläschen dicht an; es besitzt eine Länge von $9 \mathrm{~mm}(0,09 \mathrm{~mm})$, einen dorsoventralen Durchmesser von $9-10 \mathrm{~mm} \quad(0,09-0,1$ $\mathrm{mm})$ und einen mediolateralen von Horizontaler Durchschnitt des Labyrinthes von Oryzias (Stadium) 1, rechts, G. Gehirn. H'. Hörtasche. $2 \mathrm{~mm}(0,02 \mathrm{~mm})$. Nach den Stellen schwankt die Dicke des bekleidenden 
Die Fntwickelung des häutigen Labyrinthes des Knochenfisches (Oryzias latipes). 335

Epithels sehr, am dicksten ist es am vorderen und äusseren Bläschenteil, während es am hinteren und oberen am dünnsten ist. An der dicksten Stelle hat es eine Höhe von $0,01 \mathrm{~mm}$, an der dünnsten eine solche von $0,0026 \mathrm{~mm}$.

\section{Stadium 2. (84 Stunden nach Befruchtung.)}

Keine Andeutung des Riechfeldes zu sehen. Die Linsenplatte ist etwas verdickt, die Retina viel stärker pigmentiert als beim vorigen Stadium. Die Wand des Augenbläschens besteht aus mehreren Zellenschichten.

Das Hörbläschen (Tafelfig. 3, 4 und Textfig. 2) legt sich dem Hirnbläschen dicht an. Es ist seitlich noch etwas abgeplattet und hat die

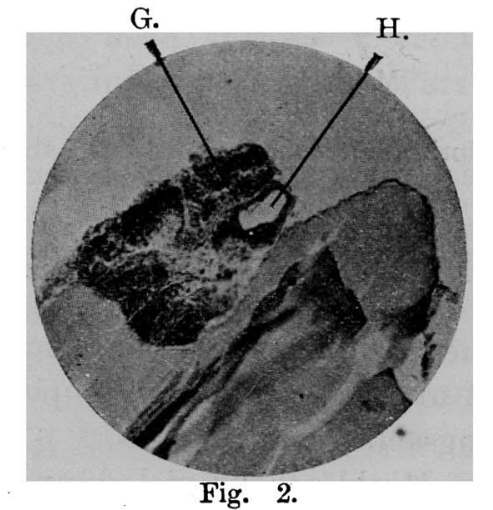

Frontaler Durchschnitt des Labyrinthes (Stadium 2, rechts, Vergr. $=$ ca. 99 mal).

G. Gehirn, H. Hörbläschen. etwas scharfen vorderen und hinteren Enden.

Auf Schnitten sieht man den Binnenraum oben etwas verschmälert und unten etwas erweitert. Das Hörbläschen besitzt einen anteroposterioren Durchmesser von $10-11 \mathrm{~mm}(0,1-0,11$ $\mathrm{mm})$, einen dorsoventralen von $9 \mathrm{~mm}$ $(0,09 \mathrm{~mm})$ und einen mediolateralen vưn $4.5 \mathrm{~mm}(0,045 \mathrm{~mm})$. Das Epithel ist an der oberen Wand niedrig und an der unteren hoch. Die ảickste Stelle besteht aus zwei Zellenschichten, während die anderen Teile aus einschichtigen Zellen sich aufbauen; an der dicksten Stelle hat es eine Höhe von $0,013-0,015 \mathrm{~mm}$, an der dünnsten die von $0,0025-0,0039 \mathrm{~mm}$.

Stadium 3. (96 Stunden nach Befruchtung.)

Das Riechfeld ist noch nicht angedeutet. Die Wand des Augenbläschens ist dick ; die Linse ist ziemlich stark verdickt, die Retina stark pigmentiert.

Das Hörbläschen (Tafelfig. 5, 6 und Textfig. 3) hat einen beträchtlichen Fortschritt der Entwickelung und Gestaltveränderung erfahren und an Volum sehr zugenommen ; alle Durchmesser sind, besonders der mediolaterale, deutlich vergrössert. Es besitzt einen anteroposterioren Durchmesser von $15 \mathrm{~mm}(0,15 \mathrm{~mm})$, einen dorsoventralen von $12 \mathrm{~mm}$ $(0,12 \mathrm{~mm})$ und einen mediolateralen von $10,5 \mathrm{~mm}(0,105 \mathrm{~mm})$. Es ist in diesem Stadium besonders im Auge zu behalten, dass hier zum ersten Male die Septumbildung für die Bogengänge zustande kommt. So findet man hier schon drei Septen und zwei Bogengänge. Das eine 
Septum dringt am vorderen oberen Teil der medialen Bläschenwand von innen (etwas oben) nach aussen (etwas unten) ein, das zweite an deren hinterem oberen Teil von hinten innen nach vorn aussen. Das dritte dringt am unteren medialen Teil der lateralen Bläschenwand von aussen (etwas hinten) nach innen (etwas vorn) ein und teilt sich in zwei Äste, von deuen der eine nach vorn oben innen eindringt und mit dem ersten medialen Septum sich verbindet, wodurch der vordere Bogengang herausmodelliert wird, während der andere nach hinten innen eindringt und mit dem zweiten medialen Septum sich verbindet, wodurch der hintere Bogengang abgeschnürt ist. Vom lateralen Bogengang ist noch keine Andeutung zu

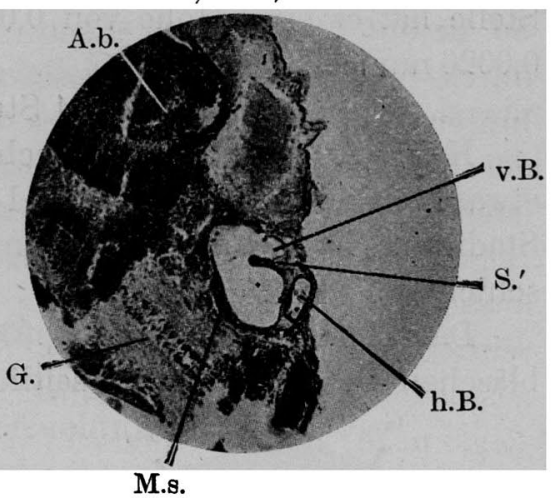

Fig. 3.

Horizontaler Durchschnitt des Labyrinthes (Stadium 3, rechts, Vergr. $=$ ca. $99 \mathrm{mal}$ )

G. Gehirn. A.b. Augenbläschen. v.B. VorG. Gehirn. A.b. Augenbläschen. v.B. Vorderer Bogengang. h.B. Hinterer Bogengang.
M.s. Macula sacculi. M.s. Macula sacculi. S' laterales $S$
den vorderen Bogengang.

sehen. Das Hörbläschen hat die Gestalt eines Hühnereies, welches mit dem spitzigen Ende nach vorn gerichtet ist und mit seinem anteroposterioren Durchmesser parallel zur Körperlängsachse steht.

Mikroskopisch ist das Wandepithel des Hörbläschens viel dünner geworden als vorher; dies beruht hauptsächlich auf der bedeutenden Erweiterung seines Binnenraumes. In diesem Stadium trifft man zum ersten Male den Beginn der Differenzierung von Nervenendstellen, was aber noch sehr geringfügig ist. So am inneren, vorderen und hinteren Teil des Bläschens ist das Epithel hoch, in der Mitte aus zwei Zellenschichten bestehend; das sind die Anlagen der Macula sacculi, Macula recessus utriculi und Crista ampullaris posterior. Das dickste Epithel ist $0,0156 \mathrm{~mm}$ hoch.

Stadium 4. (120 Stunden nach Befruchtung.)

Das Riechfeld besteht schon aus mehreren Zellenschichten. Die Augenbläschen sind äusserlich bemerkbar, die Linse stark verdickt.

Der anteroposteriore Durchmesser des Hörbläschens (Tafelfig. 7, 8 und Textfig. 4) beträgt $18 \mathrm{~mm}(0,18 \mathrm{~mm})$, der dorsoventrale $16 \mathrm{~mm}$ $(0,16 \mathrm{~mm})$ und der mediolaterale $9 \mathrm{~mm}(0,09 \mathrm{~mm})$. In diesem Stadium sieht man die erste Bildung des lateralen Bogenganges und die Differenzierung der Crista ampullaris posterior. Der vordere Bogengang zieht von vorn unten aussen nach hinten oben innen und ist seitlich stark abgeplattet, und der hintere, ebenfalls seitlich abgeplattete steigt von 
Die Entwickelung des häutigen Labyrinthes des Knochenfisches (Oryzias latipes). 337

hinten unten aussen nach vorn oben innen auf. Erst von diesem Stadium an treten die beiden Septa für den lateralen Bogengang auf. So dringt das untere Septum von unten (etwas vorn) nach oben (etwas hinten) ein und verbindet sich mit dem oberen Septum, welches vom iateralen Septum der beiden vertikalen Bogengänge sich teilt und nach unten absteigt, wodurch der laterale, auch seitlich abgeplattete Bogengang herausmodelliert wird. Das Hörbläschen zeigt noch keinen Unterschied einer Pars inferior und einer Pars superior; seine laterale Wand ist fast flach, die mediale dagegen von vorn nach hinten konvex.

Mikroskopisch. Die Isolierung der

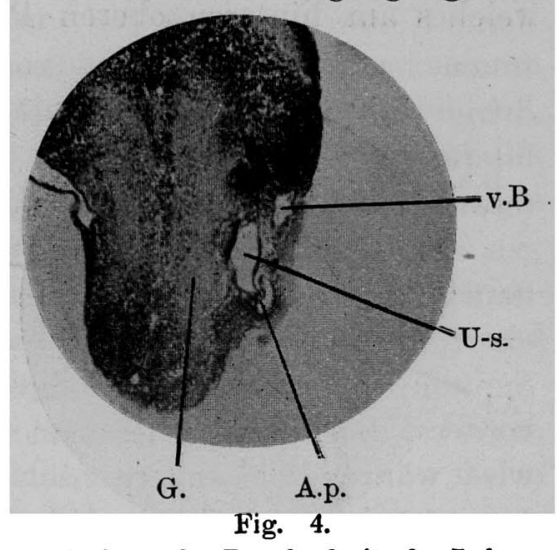

Horizontaler Durchschnitt des Labyrinthes (Stadium 4, rechts, Vergr. $=$ ca. $99 \mathrm{mal}$ ).

G. Gehirn, v.B. Vorderer Bogengang. A.p. Ampulla posterior. U-s. Utriculosacculus. Nervenendstellen ist noch nicht vervollständigt. Die Macula sacculi ist am grössten, zeigt sich auf Schnitten hügelartig und besteht an ihrer höchsten Mitte aus zwei Zellenschichten. Die Crista ampullaris posterior hat eine Halbmondform und besteht auch in der Mitte aus zwei Zellenlager. Am unteren Bläschenteil liegt die von seiner vorderen Wand bis zur unteren ziehende Macula recessus utriculi mit zweischichtigen Zellen in der Mitte. In der Mitte der unteren Bläschenwand ist der erste Beginn der Crista ampullaris lateralis zu sehen.

Stadium 5. (144 Stunden nach Befruchtung.)

Die Larve hat eine grösste Länge von $3 \mathrm{~mm}$. Das Riechfeld ist etwas tiefer geworden als vorher; die Linse ist kuglig.

Das Hörbläschen (Tafelfig. 9, 10, 11 und Textfig. 5, 6) besitzt einen anteroposterioren Durchmesser von $28,5 \mathrm{~mm}(0,285 \mathrm{~mm})$, einen dorsoventralen von $20 \mathrm{~mm}(0,2 \mathrm{~mm})$ und einen mediolateralen von $14 \mathrm{~mm}(0,14 \mathrm{~mm})$. Beachtenswert ist es, dass hier die Anlage der Ampullen gebildet und die Crista ampullaris lateralis isoliert ist. Das laterale Septum für den vorderen Bogengang dringt von aussen (etwas hinten) nach innen (etwas vorn) ein, das mediale von innen (etwas vorn) nach aussen (etwas hinten) und verbindet sich mit dem lateralen. Die äussere Wand des vorderen Bogenganges ist etwas abgeplattet. Sein vorderer Teil ist nach unten leicht gebogen, endet etwas angeschwollen und bildet den Beginn der vorderen Ampulle.

Das vom lateralen Septum des vorderen Bogenganges verzweigte 
laterale Septum für den hinteren Bogengang zieht von vorn oben aussen nach hinten unten innen und verbindet sich mit dem medialen Septum, welches am hinteren oberen Pol der inneren Hörbläschenwand von

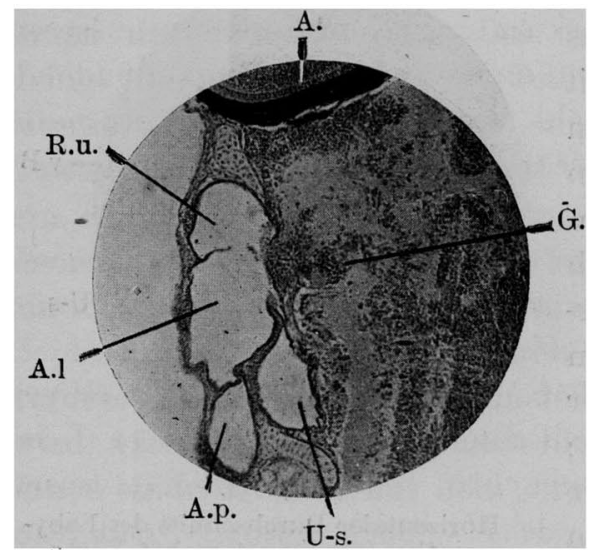

Fig. 5 .

Horizontaler Durchschn. des Labyrinthes (Stadium 5, links, Vergr. = ca. $29 \mathrm{~mm}$ ). G. Gehirn. A. Auge. A.p. Ampulla posterior. A.l. Ampulla lateralis. R.u. Recessus utriculi. U-s. Utriculosacculus.

hinten (etwas innen) nach vorn (etwas aussen) eindringt, wodurch der hintere Bogengang abgeschnürt wird. Der letztere zieht von unten hinten (etwas innen) nach oben vorn (etwas aussen) und biegt. dann stark nach innen; dieses nach innen etwas erweiterte untere Ende bildet den Béginn der Ampulla posterior. Das untere Septum des lateralen Bogenganges steigt von unten nach oben auf und schnürt den lateralen Bogengang ab, dessen vorderes Ende stark erweitert ist und die Ampulla lateralis bildet, welche viel grösser als die vordere und hintere ist. Die Ampulla lateralis trägt an ihrer lateralen Wand die Crista lateralis, welche von der Macula recessus utriculi schon weit entfernt liegt, und wird lateral vom Recessus utriculi durch eine fast senkrechte Furche abgeschieden.

Der Utriculus und Sacculus stellen miteinander einen gemeinsamen Raum, den ich den Utriculosacculus nenne, dar und ihre mediale Wand ist vorgewölbt, an deren unterem Teil die länglich-elliptische Macula sacculi liegt. Die laterale Wand geht vorn in die des Recessus utriculi, hinten in die des Sinus utriculi posterior und oben in die des Sinus utriculi superior über. Der vordere Teil des Utriculosac-

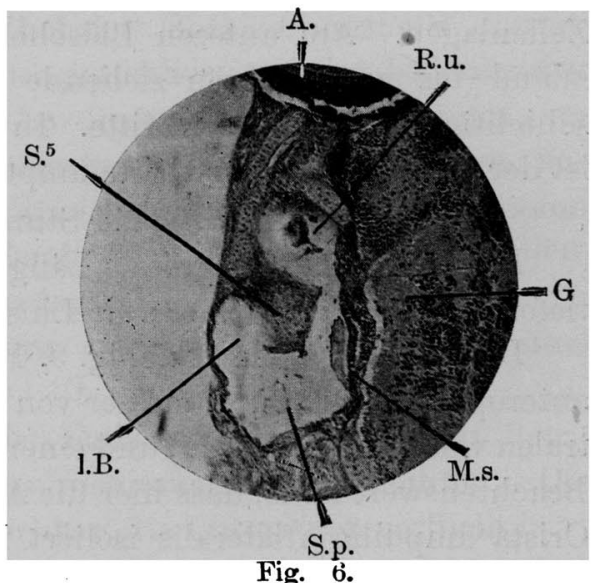

Horizontaler Durchschn. des Labyrinthes (Stadium 5, links, Vergr. = ca. $99 \mathrm{mal}$ ). G. Gehirn. 1.B. Lateraler Bogengang. R.u. Recessus utriculi. M.s. Macula sacculi. \$ $\$^{5}$ Oberes Septum für den lateralen Bogengang. S.p. Sinus utriculi posterior. A. Auge. culus, setzt sich nach vorn (etwas aussen) fort und bildet einen erweiterten Raum, der nichts anderes als der Recessus utriculi-ist, welcher nach vorn aussen in die vordere Ampulle übergeht. Auf seiner 
Die Entwickelung des häutigen Labyrinthes des Knochenfisches (Oryzias latipes). 339

vorderen lateralen Wand liegt dic Macula recessus utriculi zusammen mit der Crista ampullaris anterior. Der äussere hintere Teil des Recessus utriculi geht in die Ampulla lateralis über.

Der Sinus utriculi posterior ist kurz, ragt vom hinteren äusseren Ende des Utriculosaeculus, mit diesem einen stumpfen Winkel bildend, nach aussen hinten hervor und geht aussen oben in die Ampulla posterior, aussen unten in das hintere Ende des hinteren Bogenganges über.

Kurz und schmal ist der Sinus utriculi superior, steigt vom Utriculosacculus aufwärts und geht vorn in den vorderen Bogengang und aussen hinten in den hinteren Bogengang über.

Mikroskopisch zeigt sich die grösste Macula sacculi als einen Hügel, welcher in der Mitte aus zwei Zellenschichten besteht. Auf ihrer freien Oberfläche ruht ein mützenförmiger Otolith. Auf Schnitten erweisen die anderen Nervenendstellen, wie die Macula recessus utriculi und Crista ampullaris posterior, sich als halbmondförmig und bestehen in der Mitte aus zwei Zellenlagen. Die Crista ampullaris posterior ist grösser als beim vorigen Stadium geworden. Die Crista ampullaris lateralis ist auf Schnitten auch halbmondförmig. Von diesem Stadium an kommt das knorpelige Gewebe, die sogenannte Basalmasse von Noorden, in den Septen der Bogengänge vor.

Stadium 6. (168 Stunden nach Befruchtung, erster Tag der Ausbrütung, $4 \mathrm{~mm}$ langes Exemplar:)

Die Riechgrube ist mässig vertieft und besteht aus mehreren Zellenschichten. Andeutung der Brustflosse. Die Linse erweist sich fixiert makroskopisch als einen weissen Punkt.

Der anteroposteriore Durchmesser des Hörbläschens (Tafelfig. 12, 13, 14 und Textfig. 7,8$)$ beläuft sich auf $29 \mathrm{~mm}(0.29 \mathrm{~mm})$, der dorsoventrale auf $22 \mathrm{~mm}(0,22 \mathrm{~mm})$ und der mediolaterale auf $23 \mathrm{~mm}(0,23$ $\mathrm{mm})$; so ist seine seitliche Dicke beträchtlich vermehrt. In diesem Stadium sind alle Bestandteile des Labyrinthes gestaltet, aber ihre Ausbildung ist noch nicht vollständig. Die Ampulla anterior hat sich viel deutlicher erweitert als beim vorigen Stadium. An ihrem vorderen Pol liegt von der lateralen Wand bis zur vorderen Wand des vorderen Blindendes die gürtelförmige Crista ampullaris anterior, welche vollkommen von der Macula recessus utriculi isoliert ist. Der vordere Bogengang hat sich sehr vergrössert gegen vorher, zieht vom oberen Ende der Ampulla anterior ausgehend nach hinten oben aussen, biegt dann nach hinten. innen oben und mündet in den Sinus utriculi superior ein. Der vordere Bogengang ist seitlich abgeplattet; ihre mediale 
Wand steht weit nach oben und die laterale nach unten geneigt; dementsprechend zeigt das laterale und mediale Septum auch eine geneigte Stellung.

Die Ampulla lateralis ist tonnenförmig und grösser als die vordere

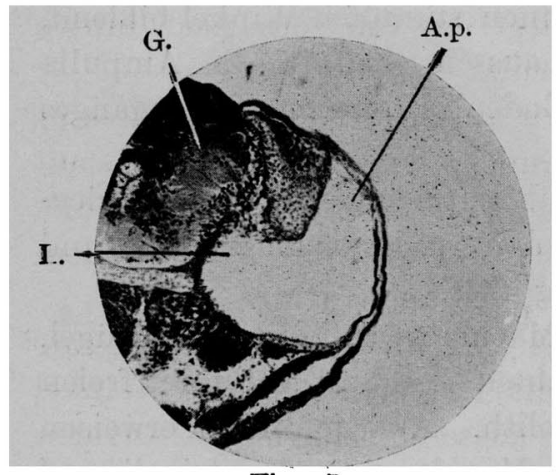

Fig. 7.

Frontaler Durchschnitt des LabyFinthes (Stadium 6, rechts, Vergr. = ca. 99 mal).

G. Gehirn. A.p. Ampulla posterior. und hintere, geht vom hinteren oberen Teil des Recessus utriculi aus und zieht horizontal nach hinten. Auf dem vorderen Teil ihrer lateralen Wand liegt die gürtelförmige Crista ampullaris lateralis, welche von vorn oben nach hinten unten verläuft. Der laterale Bogengang ist zylindrisch und kürzer als die Ampulla lateralis, geht von der letzteren aus, zieht nach hinten (etwas innen) und mündet in den Sinus utriculi posterior aus. Die Ampulla posterior befindet sich aussen oben vom Sinus utriculi posterior und vorn von der Ausmündungsstelle des lateralen Bogenganges in den letzteren. Sie geht oben vorn ohne scharfe Grenze in den hinteren Bogengang über. Die Crista ampullaris posterior liegt die hintere Wand der Ampulla horizontal nach hinten aussen umgürtend. Der hintere Bogengang ist an Volumen viel grösser als der vordere und laterale, geht von der Ampulla posterior aus, biegt sich nach vorn oben innen und mündet in den äusseren, oberen Teil des Sinus utriculi superior ein, indem er mit dem vorderen Bogengang einen nach aussen offenen, mehr spitzigen Winkel bildet. Das laterale Septum für den hinteren Bogengang schreitet von aussen vorn nach innen hinten und verbindet

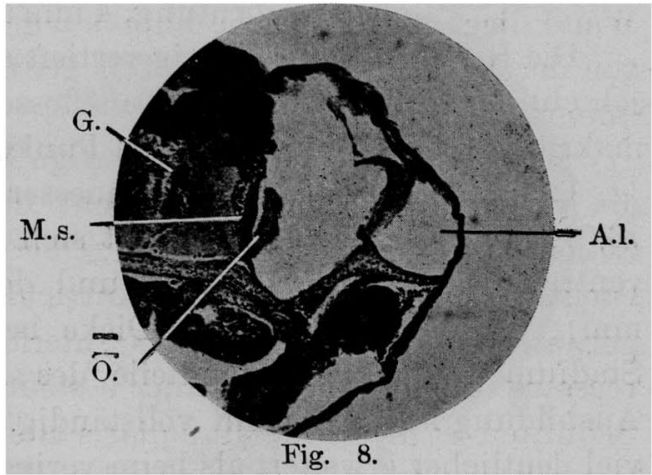

Frontaler Durchschnitt des Labyrinthes (Stadium 6, rechts, Vergr. = ca. 99 mal). G. Gehirn. A.l. Ampulla lateralis. M.s. Macula sacculi. O. Otolith. sich mit dem medialen, welches von hinten innen nach vorn aussen eindringt.

Der tonnenförmige Utriculosacculus ist von vorn nach hinten verlängert, seine vordere obere Wand steht stark geneigt und fasst zwischen sich und dem unteren Rand des vorderen Bogenganges eine 
Die Entwickelung des häutigen Labyrinthes des Knochenfisches (Oryzias latipes). 341

Spalte ein. Nach vorne allmählich sich verschmälernd, geht er in den Recessus utriculi über, wovon er aber durch eine deutliche ringförmige Furche abgegrenzt ist; hinten geht er in den Sinus utriculi posterior, oben ohne scharfe Grenze in den kurzen Sinus utriculi superior über. Auf der Mitte seiner medialen Wand liegt die von vorn nach hinten verlaufende grosse elliptische Macula sacculi.

Der vom Utriculosacculus nach vorn aussen ausgehende Recessus utriculi stellt einen von aussen nach innen verlängerten Raum mit schalenförmigem Boden dar. Vorn setzt er sich in die Ampulla anterior, aussen hinten in die Ampulla lateralis fort. Auf seinem Boden befindet sich die rundliche Macula recessus utriculi.

Der vom hinteren unteren $\mathrm{Pol}$ des Utriculosacculus ausgehende Sinus utriculi posterior zieht, mit diesem fast einen rechten Winkel bildend, nach aussen und geht mit seinem äusseren Ende in den lateralen Bogengang, mit dem oberen äusseren Ende in die Ampulla posterior über.

Der Sinus utrieuli superior ist kurz und steigt vom hinteren oberen Teil des Utriculosacculus nach oben auf; vorn setzt er in das hintere Ende des vorderen Bogenganges, aussen oben in das vordere Ende des hinteren Bogenganges über.

Die Lagena cochleae ist kurz, halbkuglig und zieht vom hinteren Ende des Utriculosacculus ausgehend nach innen. Auf ihrer medialen Wand liegt die rundliche Papilla lagenae, welche auf der linken Seite von der Macula sacculi zum Teil getrennt, auf der rechten noch nicht isoliert ist.

Mikroskopisch ist die hügelartige Macula sacculi grösser und höher als vorher geworden und besteht in der Mitte aus zweischichtigen Zellenlagen. Auf ihrer freien Oberfläche ruht ein mützenförmiger Otolith. Die Macula recessus utriculi ist der Macula sacculi ähnlich gebaut. Die Crista ampullaris anterior ist vollständig von der Macula recessus utriculi isoliert und besteht aus zwei Zellenschichten. Auf Schnitten ist die Crista ampullaris lateralis halbmondförmig, die Crista ampullaris posterior zeigt sich bei Flächenansicht gürtelförmig. Von der Macula sacculi zum Teil isoliert ist die Papilla lagenae mit der hohen Epithèlzone noch damit zusammenhängend. Die Basalmasse hat sich gegen das vorige Stadium bedeutend vermehrt.

Stadium 7. (8 Tage nach Ausbrütung, $7 \mathrm{~mm}$ langes Exemplar.)

Die Riechgrube ist äusserst vertieft.

Das Labyrinth (Tafelfig. 15, 16, 17 und Textfig. 9) besitzt einen anteroposterioren Durchmesser von $36,6 \mathrm{~mm}(0,555 \mathrm{~mm})$, einen dorso- 
ventralen von $23 \mathrm{~mm}(0,348 \mathrm{~mm})$ und einen mediolateralen von $22 \mathrm{~mm}$ $(0,332 \mathrm{~mm})$.

Die halbkuglige Ampulla anterior geht vom vorderen Ende des -Recessus utriculi aus, steigt nach vorn oben auf und geht nach hinten oben sich verschmälernd in den vorderen Bogengang über. Von ihrer äusseren Wand bis zur vorderen verläuft die gürtelförmige Crista ampullaris anterior. Der von Ampulla anterior nach hinten oben innen ausgehende, leichtgebogene vordere Bogengang mündet in das vordere Ende des Sinus utriculi superior ein. Seine mediale Wand ist noch

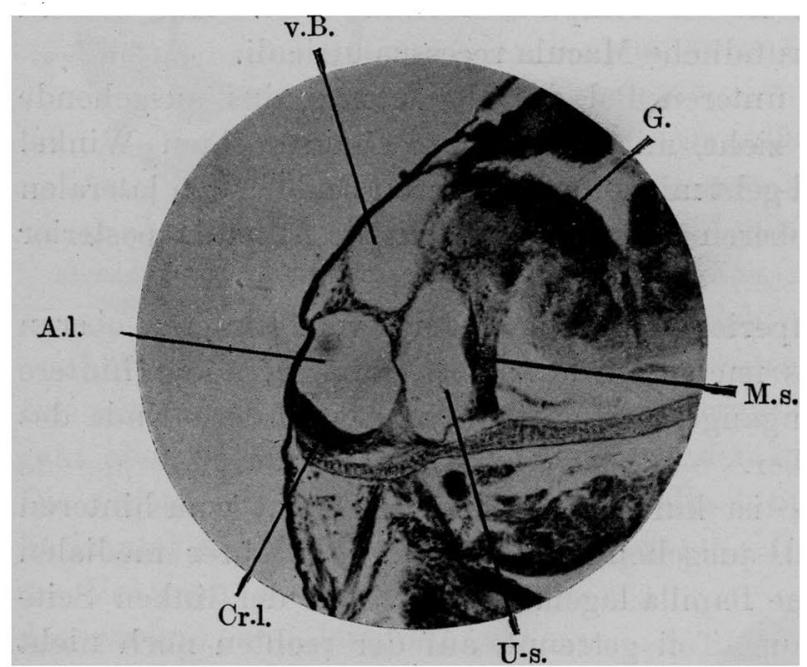

Fig. 9. (Stadium 7, links, Vergr.= ca. 99 mal.)

G. Gehirn. v.B. Vorderer Bogengang.

$\begin{array}{ll}\text { A.l. Ampulia lateralis. } & \text { U-s. Utriculosacculus. } \\ \text { M.s. Macula sacculi. } & \text { Cr.i. Crista ampullaris lateralis. }\end{array}$
Frontaler Durchschnitt des Labyrinthes

flach, während die laterale etwas angeschwollen ist.

Die Ampulla lateralis ist viel grösser als die Ampulla anterior und stellt einen horizontal liegenden Zylinder dar. Sie geht vom hinteren äusseren oberen Teil des Recessus utriculi aus, zieht nach hinten aussen und ist vom Recessus utriculi mit einer seichten ringförmigen Furche abgegrenzt. Ihr hinteres Ende verschmälert sich und geht in den lateralen Bogengang über. Auf ihrer lateralen Wand liegt vorn die von oben nach unten verlaufende gürtelförmige Crista ampullaris lateralis. Der laterale Bogengang zieht zuerst nach hinten unten, biegt dann nach hinten oben und mündet in den Sinus utriculi posterior ein. Er ist noch viel kürzer als die zu ihm gehörige Ampulla.

Die aussen oben vom Sinus utriculi posterior ausgehende Ampulla posterior zieht nach oben und ihre mediale Wand ist etwas abgeplattet. Oben etwas verschmälert, geht sie in den hinteren Bogengang über. Auf ihrer lateralen Wand liegt die Crista ampullaris posterior, welche vom hinteren Teil der lateralen Wand bis zur hinteren Wand verläuft. Der hintere Bogengang geht vom oberen Ende der Ampulla posterior aus, schreitet nach oben vorn und biegt sich nach oben innen, um end- 
Die Entwickelung des häutigen Labyrinthes des Knochenfisches (Oryzias latipes). 343

lich in das hintere äussere Ende des Sinus utriculi superior überzugehen.

Der Utriculosacculus weist einen eimheitlichen Raum auf und ist von vorn nach hinten verlängert. Seine mediale Wand ist abgeplattet und trägt die anteroposterior längliche elliptische Macula sacculi; die laterale Wand ist etwas aufgetrieben. Vorn oben geht er in den Recessus utriculi, hinten in den Sinus utriculi posterior sowie in die Lagena cochleae und oben in den Sinus utriculi superior über.

Der vom Utriculosacculus nach vorn unten ausgehende Recessus utriculi ist durch eine schwache ringförmige Furche vom Utriculosacculus abgegrenzt; er nimmt vorn oben die Ampulla anterior, hinten aussen oben die Ampulla lateralis auf. Auf seinem konkaven Boden liegt die elliptische Macula recessus utriculi.

Der vom hinteren äusseren Ende des Utriculosacculus ausgehende Sinus utriculi posterior ist kurz, knickt sich stark nach aussen (etwas hinten) und geht aussen vorn unten in den lateralen Bogengang, oben aussen in die Ampulla posterior über.

Der Sinus utriculi superior steigt vom Utriculosacculus nach oben empor und kommuniziert vorn mit dem vorderen, hinten aussen mit dem hinteren Bogengang.

Die Lagena cochleae ist halbkuglig, geht vom hinteren inneren Ende des Utriculosacculus aus und läuft nach innen; auf ihrer medialen Wand liegt die rundliche Papilla lagenae, welche von der Macula sacculi jetzt schon vollständig losgetrennt ist.

Mikroskopisch zeigt sich die Macula sacculi auf senkrechten Schnitten als einen Hügel und besteht in der Mitte aus zwej Zellenschichten. Die Macula recessus utriculi ist der Macula sacculi ähnlich gebaut. Bei Flächenansicht erweisen sich die Crista ampullaris anterior und posterior als gürtelförmig und die Crista ampullaris lateralis auf Schnitten als halbmondförmig. Die Papilla lagenae hat auf Schnitten eine hügelartige Form und setzt sich in der Mitte auch aus zweischichtig gelagerten Zellen zusammen.

Stadium 8. (15 Tage nach Ausbrütung, $9 \mathrm{~mm}$ langes

Exemplar (Kopflänge etwa $2 \mathrm{~mm}$.))

Der anteroposteriore Durchmesser des häutigen Labyrinthes (Tafelfig. 18, 19, 20 und Textfig. 10) beläuft sich auf $32 \mathrm{~mm}(0,64 \mathrm{~mm})$, der dorsoventrale auf $31 \mathrm{~mm}(0,52 \mathrm{~mm})$ und der mediolaterale auf $24 \mathrm{~mm}$ $(0,48 \mathrm{~mm})$.

Die Ampulla anterior ist kurz, kuglig, geht vom vorderen oberen Ende des Recessus utriculi aus und zieht nach vorn oben; ihre Grenze gegen den Recessus utriculi ist undeutlich. Nach vorne allmählich sich 
verschmälernd, geht dieser in den vorderen Bogengang über, welcher von der Ampulla anterior nach oben aufsteigt, dann nach hinten oben innen zieht und in den Sinus utriculi superior einmündet. Auf seinem konkaven Boden liegt die von vorn innen unten nach hinten aussen oben verlaufende Crista ampullaris anterior.

Die vom oberen Teil des Recessus utriculi ausgehende Ampulla lateralis ist ein länglich-rundes Rohr, zieht erst nach hinten und etwas aussen, biegt dann nach hinten und etwas unten, und geht endlich von medial verengt, in den lateralen Bogengang über. Zwischen dem Recessus utriculi und der Ampulla lateralis findet man eine ringförmige Furche, welche viel deutlicher ausgeprägt ist als beim vorigen Stadium.

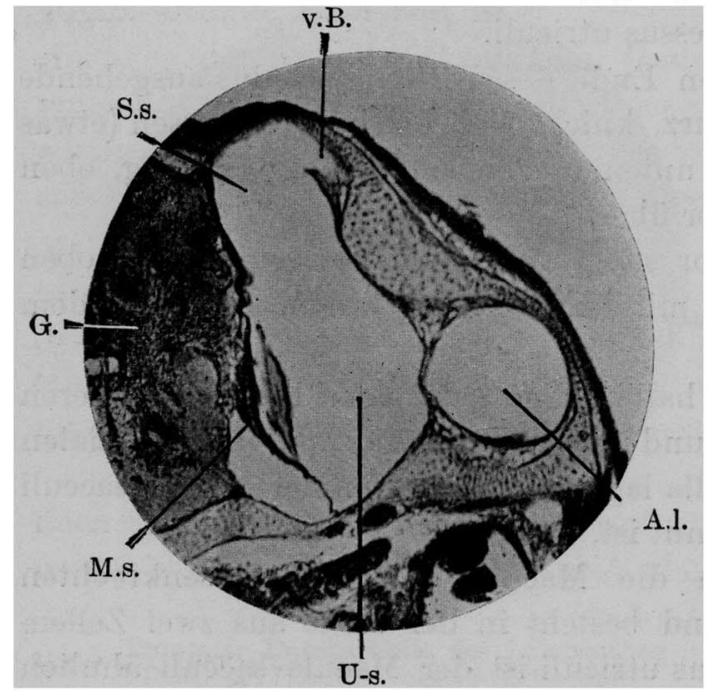

Fig. 10 .

Frontaler Durchschnitt des Labyrinthes

(Stadium 8, rechts, Vergr. = ca. 78 mal.)

v.B. Vorderer Bogengang. A.l. Ampulla lateralis.

S.s. Sinus utriculi superior. U-s. Utriculosacculus. Auf der lateralen Wand der Ampulle liegt in der Mitte die von oben aussen nach innen unten verlaufende gürtelförmige Crista ampullaris lateralis. Diese Ampulle liegt in der gleichen horizontalen Höhe wie die Ampulla anterior, ist jedoch viel grösser als diese. Der laterale Bogengang ist zylindrisch, gebogen und viel länger als beim vorigen Stadium; er geht vom hinteren Ende der. Ampulla lateralis, zieht zuerst nach hinten aussen und dann nach hinten (etwas oben), um endlich nach hinten innen biegend in den Sinus utriculi posterior überzugehen.

Die kuglige Ampulla posterior geht vom äusseren oberen Ende des Sinus utriculi posterior aus und nach oben sich verschmälernd in den hinteren Bogengang über. Auf ihrer hinteren Wand liegt die von aussen nach innen verlaufende gürtelförmige Crista ampullaris posterior. Der hintere Bogengang geht von der Ampulla posterior aus, zieht nach vorn oben, biegt sich dann stark nach innen und mündet in den Sinus utriculi superior ein. Dieser ist viel deutlicher zylindrisch als beim vorigen Stadium.

Die mediale und laterale Wand des Utriculosacculus sind halb- 
Die Entwickelung des häutigen Labyrinthes des Knochenfisches (Oryzias latipes). 345

kuglig angeschwollen; der untere Teil der lateralen Wand bildet stark nach innen unten sich verschiebend mit der medialen Wand zusammen eine von vorn nach hinten verlaufende untere Kante. Nach vorn geht der Utriculosacculus in den Recessus utriculi, nach binten innen in die Lagena cochleae, nach hinten aussen in den Sinus utriculi posterior und nach oben in den Sinus utriculi superior über. In der Mitte seiner medialen Wand findet man die anteroposterior verlängerte elliptische Macula sacculi.

Der Recessus utriculi ragt vom vorderen Ende des Utriculosacculus nach vorn unten (etwas aussen) hervor und ist durch eine deutliche Einschnürung von diesem abgegrenzt. Sein Boden ist abwärts kuglig vorgewölbt, das Dach dagegen etwas konkav. Nach vorn geht er in die Ampulla anterior und hinten aussen in die Ampulla lateralis, mit einer ringförmigen Furche begrenzt, über. In der Mitte des Bodens liegt die von vorn innen nach hinten aussen neigende elliptische Macula recessus utriculi.

Der Sinus utriculi posterior ist kurz-zylindrisch und ragt vom äusseren hinteren Ende des Utriculosacculus nach aussen (etwas hinten) einen starken Winkel bildend hervor; nach aussen oben geht er in die Ampulla posterior und nach aussen hinten in den lateralen Bogengang über.

Der Sinus utriculi superior ist kurz, steigt vom Utriculosacculus nach oben empor und setzt sich nach vorn in den vorderen, nach aussen hinten in den hinteren Bogengang fort.

Die Lagena cochleae ist halbkuglig und ragt vom hinteren inneren Ende des Utriculosacculus nach innen hinten hervor. Sie verbindet sich mit dem letzteren durch ein rundliches grosses Loch. Auf ihrer medialen Wand erkennt man die rundliche Papilla lagenae.

Mikroskopisch: Die Macula sacculi ist die grösste von allen Nervenendstellen, zeigt sich auf senkrechten Schnitten als einen Hügel und besteht aus zwei Zellenschichten. Auf ihrer freien Oberfläche ruht der Otolith. Die Macula recessus utriculi und Papilla lagenae sind der Macula sacculi ganz ähnlich zusammengesetzt, obgleich die letztere keinen Otolith trägt. Bei Flächenansicht sind die Crista ampullaris anterior und posterior beide gürtelförmig und die Crista ampullaris lateralis erweist sich auf Schnitten als halbmondförmig. Erst von diesem Stadium an kommt das Septum nervum vor, welches bei der Ampulla anterior von der unteren Wand, bei der Ampulla lateralis und posterior von der lateralen Wand in den Binnenraum der Ampullen 
hineinragt. Die Basalmasse ist hier schon zum Knorpelgewebe umgewandelt.

Stadium 9. (35 Tage nach Ausbrütung, $13 \mathrm{~mm}$ langes Exemplar (Kopflänge etwa $3 \mathrm{~mm}$.))

Das häutige Labyrinth (Tafelfig. 21, 22, 23 und Textfig. 11) besitzt einen anteroposterioren Durchmesser von $49 \mathrm{~mm}(0,98 \mathrm{~mm})$, einen dorsoventralen von $30 \mathrm{~mm}(0,6 \mathrm{~mm})$ und einen mediolateralen von $34 \mathrm{~mm}$ $(0,68 \mathrm{~mm})$. Alle seine Abteilungen sind gut ausgebildet und denen des ausgewachsenen Tieres fast gleich.

Die vom vorderen Teil des Recessus utriculi nach vorn oben (etwas innen) ausgehende kuglige Ampulla anterior ist durch eine sehr schwache ringförmige Einschnürung von dem ersteren abgegrenzt. Sie zieht zuerst nach vorn oben, biegt sich dann nach vorn innen und geht in den vorderen Bogengang über. Auf ihrer lateralen Wand liegt das von vorn innen unten nach hinten aussen oben laufende Septum nervum; auf diesem ruht die gürtelförmige Crista ampullaris anterior. Der vordere Bogengang zieht von der Ampulla anterior entspringend nach hinten oben

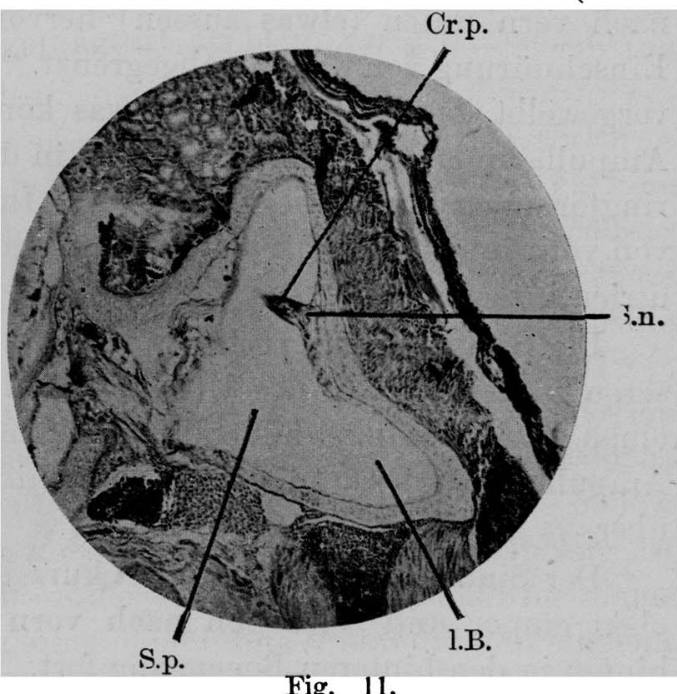
Fig. 11 .

Frontaler Durchschnitt des Labyrinthes (Stadium 9, links, Vergr. = ca. 78 mal.)

1.B. Lateraler Bogengang. S.p. Sinus utriculi posterior. 1.B. Lateraler Bogengang. S.p. Sinus utriculi posterior.
s.n. Septum nervum. innen, biegt sich dann etwas nach innen und mündet in den Sinus atriculi superior ein. Die mediale Wand des Bogenganges ist abgeplattet; je näher der letztere dem Sinus utriculi superior kommt, desto mehr verlängert sich sein dorsoventraler Durchmesser und wird der Gang seitlich platter.

Die vom oberen Teil des Recessus utriculi ausgehende Ampulla lateralis repräsentiert ein länglich rundes Rohr und ist von vorn oben nach hinten aussen etwas umgedreht; zuerst zieht sie nach hinten oben, biegt sich dann nach hinten aussen (etwas unten) und geht endlich in den lateralen Bogengang über. Sie nimmt ihre Lage viel höher ein und ist viel grösser als die Ampulla anterior. Zwischen ihr und dem Recessus utriculi befindet sich eine ringförmige Furche, welche an der lateralen 
Die Entwickelung des häutigen Labyrinthes des Knochenfisches (Oryzias latipes). 347

Wand deutlicher als an der medialen ist. Auf ihrer lateralen Wand vorn liegt das von vorn oben nach hinten unten verlaufende Septum nervum; auf diesem sitzt die gürtelförmige Crista ampullaris lateralis. Der laterale Bogengang weist ein gebogenes zylindrisches Rohr auf und entspringt vom hinteren äusseren Ende der Ampulla lateralis. Er zieht nach hinten aussen, biegt sich dann nach hinten innen und mündet in das hintere äussere Ende des Sinus utriculi posterior ein.

Die Ampulla posterior steigt vom Dach des Sinus utriculi posterior nach oben, zieht nach vorn oben und geht in den hinteren Bogengang über. Zwischen ibr und dem Sinus sieht man eine Furche, welche nur auf der lateralen Wand kaum erkennbar ist. Auf der hinteren Wand der Ampulle liegt das von aussen nach medial verlaufende Septum nervum, auf welchem die gürtelförmige Crista ampullaris posterior aufsitzt. Der hintere Bogengang entspringt vom oberen Ende der Ampulla posterior, steigt zuerst nach oben, zieht dann nach vorn oben innen und biegt sich endlich stark nach innen, um in den Sinus utriculi superior einzumünden.

Der Utriculosacculus geht vorn in den Recessus atriculi, hinten aussen in den Sinus utriculi posterior und hinten innen in die Lagena cochleae über. Seine mediale und laterale Wand sind kuglig angeschwollen. Die Einschnürung zwischen diesem und dem Recessus utriculi ist viel deutlicher ausgeprägt als beim vorigen Stadium. In der Mitte seiner medialen Wand liegt die elliptische grosse Macula sacculi. Die mediale und laterale Wand bilden miteinander verbunden ventral eine von vorn nach hinten verlaufende Kante. Die vorderen Teile der medialen und lateralen Wand sind verschmälert und flach.

Der Recessus utriculi entspringt vom Utriculosacculus, zieht nach vorn aussen, steht von aussen oben nach innen unten geneigt und ist durch eine deutliche Einschnürung von diesem abgegrenzt; er hat ein flaches Dach und einen kugligen Boden, auf welch letzterem die elliptische Macula recessus utriculi sich befindet. Vorn geht er in die Ampulla anterior, aussen oben in die Ampulla lateralis über.

Der Sinus utriculi posterior ist kurz, entspringt rechtwinklig vom hinteren unteren äusseren Ende des Utriculosacculus, zieht nach aussen und geht aussen in den lateralen Bogengang, oben in die Ampulla posterior über.

Vom Utriculosacculus entspringt nach oben der kurze Sinus utriculi superior, welcher vorn in den vorderen, aussen oben in den lateralen Bogengang übergeht.

Die Lagena cochleae ist eine kuglige Blase, geht vom hinteren 
inneren unteren Ende des Utriculosacculus aus, zieht nach hinten innen und mündet durch ein grosses rundes Loch in den Utriculosacculus ein. Auf ihrer medialen Wand liegt die rundliche Papilla lagenae.

Mikroskopisch. Die Crista ampullaris anterior und posterior erweisen sich bei Flächenansicht als gürtelförmig und die Crista ampullaris lateralis auf Schnitten als halbmondförmig. Die Macula sacculi und Macula recessus utriculi zeigen auf Schnitten eine Hügelform; auf deren freien Oberfläche ruht der mützenförmige Otolith. Das Septum nervum ist viel deutlicher ausgebildet als beim vorigen Stadium.

Stadium 10. (34 mm langes ausgewachsenes Exemplar

(Kopflänge etwa $7 \mathrm{~mm}$.$) )$

Das häutige Labyrinth (Tafelfig. 24, 25, 26 und Textfig. 12, 13, 14) besitzt einen anteroposterioren Durchmesser von $77,2 \mathrm{~mm}(2,34 \mathrm{~mm})$,

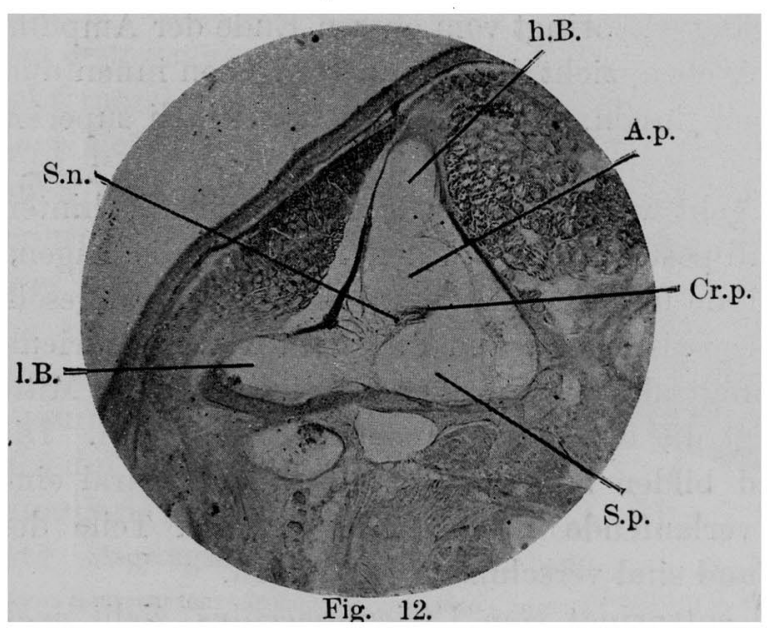

Frontaler Durchschnitt des Labyrinthes (Stadium 10, links, Vergr.=ca. 27.5 mal).

l.B. Lateraler Bogengang. A.p. Ampulla posterior.

S.n. Septum nervum. $\quad$ Cr.p. Crista amp. posterior.

S.p. Sinus utr. posterior. h.B. Hinterer Bogengang einen dorsoventralen von $49 \mathrm{~mm}(1,48 \mathrm{~mm})$ und einen mediolateralen von $59,4 \mathrm{~mm}(1$, $8 \mathrm{~mm})$.

Die Ampulla anterior hat kuglige Form und entspringt vom vorderen oberen Ende des Recessus utriculi; sie steigt nach vorn oben, dann nach vorn oben aussen und setzt sich in den vorderen Bogengang fort. Sie ist durch eine schwache Einschnürung, welche medial tief und lateral seicht ist, vom Recessus utriculi abgegrenzt. Auf ihrer lateralen Wand liegt das von vorn innen nach hinten aussen ziehende Septum nervum, worauf die gürtelförmige Crista ampullaris anterior aufsitzt. Der vordere Bogengang hat eine Länge von $31 \mathrm{~mm}(1,24$ $\mathrm{mm}$ ) und ist leicht gebogen; er entspringt von der Ampulla anterior, zieht nach oben, dann nach hinten innen oben und mündet in den Sinus utriculi superior ein. Sein vorderer Teil ist zylindrisch, je weiter er aber nach hinten kommt, desto grösser wird seîn mediolateraler Durchmesser, wodurch er, sich dorsoventral verkürzend, horizontal sehr flach wird.

Die Ampulla lateralis ist tonnenförmig, entspringt vom oberen 
Die Entwickelung des häutigen Labyrinthes des Knochenfisches (Oryzias latipes). 349

Ende des Recessus utriculi, zieht von vorn inmen nach hinten aussen und geht in den lateralen Bogengang über. Sie hat die höchste horizontale Lage und ist auch am grössten unter allen drei Ampullen. Ihr vorderer äusserer Teil liegt unter dem vorderen Bogengang verborgen. Zwischen ihr und dem Recessus utriculi liegt eine ringförmige Furche, welche an der äusseren hinteren und vorderen Seite tief und an der inneren sehr seicht ist. Auf ihrer medialen Wand befindet sich das bogenförmig von oben nach unten ziehende Septum nervum, auf welchem die gürtelförmige Crista ampullaris lateralis sitzt. Der laterale Bogengang hat eine Länge von $47 \mathrm{~mm}(1,424 \mathrm{~mm})$, ist lang und schmal und stellt ein horizontales bogenförmiges Rohr dar; er ist der schmälste und längste von allen drei Bogengängen und kommuniziert mit dem äusseren unteren Teil der Ampulla posterior und dem Sinus utriculi posterior. Er ist vom Utriculosacculus viel weiter entfernt gelegen als beim vorigen Stadium.

Die Ampulla posterior liegt auch vom Utriculosacculus viel weiter enfernt als vorher und

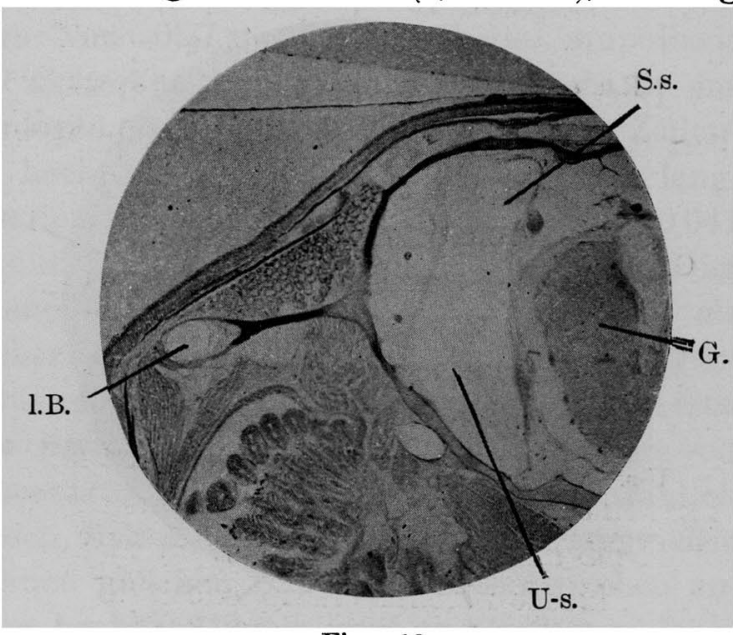

Fig. 13.

Frontaler Durchschnitt des Labyrinthes (Stadium 10, links, Vergr.= ca. 21. mal).

I.B. Lateraler Bogengang. S.s. Sinus utr. superior. U-s. Utriculosacculus. G, Gehirn.

entspringt vom hinteren oberen Teil des Sinus utriculi posterior; sie läuft nach oben hinten aussen und dann nach oben innen, um zuletzt in den lateralen Bogengang überzugehen. Auf ihrer medialen Wand liegt in der Mitte das zuerst von vorn nach hinten, dann nach innen zichende Septum nervum, das die gürtelförmige Crista ampullaris posterior trägt. Die Ampulla hat eine ähnliche Gestalt wie die Ampulla anterior, steht aber etwas höher als diese. Der hintere Bogengang hat eine Länge von $25,7 \mathrm{~mm}(0,78 \mathrm{~mm})$ und ist zylindrisch, entspringt vom unteren äusseren oberen Ende der Ampulla posterior, steigt nach aussen oben (etwas vorn), biegt sich dann nach vorn oben innen und ist von medial nach lateral erweitert; er mündet in den Sinus utriculi superior ein. 
Der Utriculosacculus hat eine besondere Form wie bei den übrigen Teleostiern, sodass er einen ganz einheitlichen Raum darstellt; er zeigt keine Andeutung der Teilung in einen Utriculus und einen Sacculus. Seine mediale Wand bildet eine senkrechte Ebene, neigt vorn nach innen vorn und bildet mit der lateralen Wand einen seitlich abgeplatteten Kanal, welcher sich vorn in den Recessus utriculi fortsetzt. Die laterale Wand ist kuglig vorgewölbt und bildet mit der medialen sich verbindend eine scharfe ventrale Kante. Der obere Rand seines vorderen Teils neigt sich von hinten oben nach vorn unten. Das vordere Ende jener

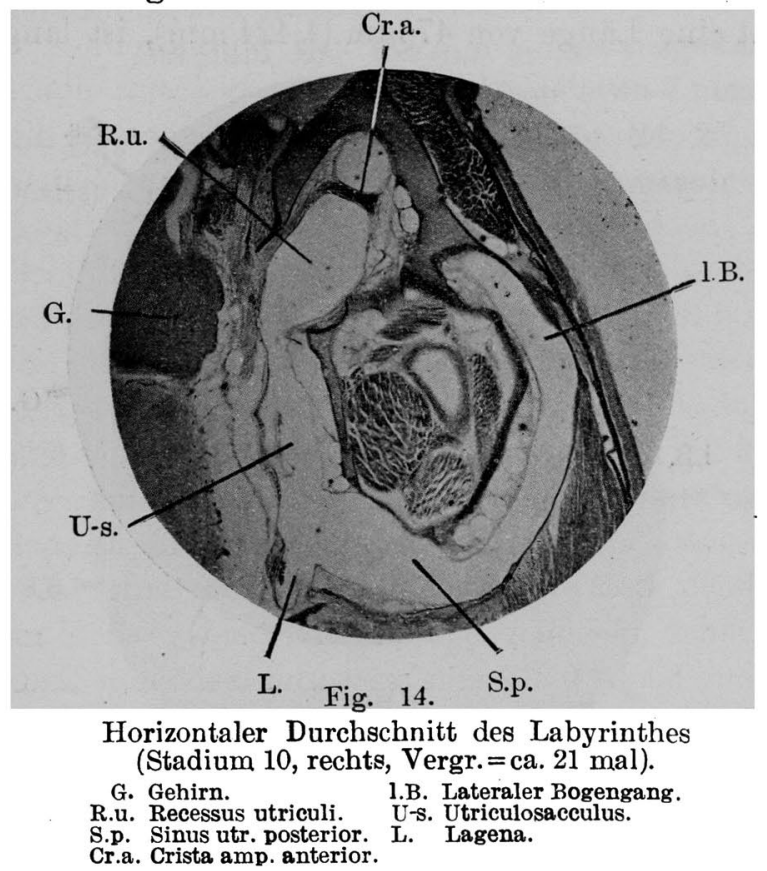
Kante steigt plötzlich nach vorn oben. Seine hintere Wand stellt eine senkrechte Ebene dar und ist am unteren Teil etwas erweitert. Er geht hier aussen in den Sinus utriculi posterior, innen in die Lagena cochleae über. Sein oberer Teil setzt sich in den Sinus utriculi superior fort. Auf seiner medialen Wand liegt in der Mitte die horizontal sehr verlängerte elliptische Macula sacculi.

Der vom Utriculosacculus nach vorn aussen sich fortsetzende Recessus utriculi hat ein ebenes, nach aussen oben geneigtes Dach und einen kugligen Boden, worauf in der Mitte die von vorn (etwas innen) nach hinten (etwas aussen) verlaufende länglich-runde Macula recessus utriculi liegt. Nach vorn aussen geht er in die Ampulla anterior, aussen oben in die Ampulla lateralis über.

Der Sinus utriculi posterior ist zylindrisch und viel bedeutender verlängert als beim vorigen Stadium, geht vom hinteren äusseren Teil des Utriculosacculus mit einem stumpfen Winkel aus und zieht nach hinten aussen ; nach hinten aussen geht er in den lateralen Bogengang, nach aussen oben in die Ampulla posterior über.

Der Sinus utriculi superior ist kurz und weit und geht nach oben vorn in den vorderen Bogengang, nach hinten aussen in den hinteren 
Die Entwickelung des häutigen Labyrinthes des Knochenfisches (Oryzias latipes). 351

Bogengang über. Seine obere Wand ist flach und weist keinen Apex auf.

Die Lagena cochleae ist kuglig und ragt vom hinteren unteren inneren Ende des Utriculosacculus nach hinten innen hervor, womit sie durch ein grosses rundes Loch kommuniziert. Ihre mediale Wand ist etwas angeschwollen und trägt die vertikale elliptische Papilla lagenae.

Mikroskopisch zeigt die Crista ampullaris anterior bei Flächenansicht eine Gürtelform, besteht in der Mitte aus zwei und peripher nur aus einer Zellenschicht, die in das Deckepithel der Ampullenwand übergeht. Die Crista hat eine Höhe von $0,055 \mathrm{~mm}$, eine Breite von $0,124 \mathrm{~mm}$ und eine Länge von $0,27 \mathrm{~mm}$. Die Crista ampullaris lateralis ist auf senkrechten Schnitten halbmondförmig, besteht in der Mitte auch aus zwei Zellenlagen und peripher nur aus einer Zellenschicht; sie ist $0,047 \mathrm{~mm}$ hoch, $0,15 \mathrm{~mm}$ breit und $0,246 \mathrm{~mm}$ lang. Die Crista ampullaris posterior ist der vorderen ähnlich gebaut, 0,041 $\mathrm{mm}$ hoch, $0,123 \mathrm{~mm}$ breit uud 0,24 mm lang. Die Macula sacculi ist die grösste von allen Nervenendstellen, erweist sich auf Schnitten als einen niedrigen Hügel, welcher in der Mitte aus zweischichtig gelagerten Zellen und peripher nur aus einer Zellenschicht besteht. Sie hat eine Höhe von $0.0275 \mathrm{~mm}$, eine Breite von $0,55 \mathrm{~mm}$ und eine Länge von $0,726 \mathrm{~mm}$. Die Macula recessus utriculi ist der Macula sacculi ähnlich aufgebaut und $0,0344 \mathrm{~mm}$ hoch, $0,39 \mathrm{~mm}$ breit und $0,48 \mathrm{~mm}$ lang. Die Papilla lagenae hat auch einen gleichen Bau wie die beiden eben angegebenen und ist $0,025 \mathrm{~mm}$ hoch, $0,312 \mathrm{~mm}$ breit und $0,455 \mathrm{~mm}$ lang.

Die tiefe Zellenlage des Neuroepithels legt der Lamina propria sich dicht an und hat im allgemeinen spindelförmige oder länglich-runde Kerne in der Nähe der Zellbasis. Die Zellen der oberflächlichen Schicht sind flaschenförmig, fein granuliert und enthalten in ihrer Mitte einen spindelförmigen Kern. Die freie Oberfläche der Zellen ist mit einer schwarzen Linie, Membrana limitans, versehen.

\section{Vergleichung und Zusammenfassung.}

\section{Das Hörbläschen.}

Nacb Krause ('06) betrifft bei denjenigen Wirbeltieren, welche ein zweischichtiges Ektoderm besitzen, wie Teleostiern, Ganoiden und Amphibien, die Epithelverdickung und Einsenkung ausschliesslich das innere Blatt, während die Deckschicht unverändert die Hörplatte und später über die Hörgrube ohne jede Einsenkung hinwegzieht. Wenn sich das Hörbläschen vollständig von seinem Mutterboden, der Epider- 
mis, abgeschnürt hat, stellt es ein länglich-ovales Bläschen dar, dessen grösster Durchmesser bei Amphibien, Vögeln und Säugetieren dorsoventral; bei Fischen und Reptilien dagegen kaudozerebral gelagert ist. Noorden ('83)'sagt bei Knochenfischen : ,, Im Verlauf der weiteren Stadien stülpt sich eine Tasche (Hörtasche) an dieser Stelle ein, welche nach ihrer Anlage zunächst die Form einer längsgestellten Spalte annimmt. Bei Eiern, die bei $7^{\circ} \mathrm{C}$. zur Entwickelung gebracht wurden, hat sich diese Tasche 48 Stunden nach Befruchtung zu einer Blase abgeschnürt. Ihre Epithelauskleidung ist zunächst sehr dünn und besteht aus einschichtig gelagerten Zellen. Die Epithelien nehmen bald an Höhe zu, ohne ihre Einschichtigkeit aufzugeben. 'Zugleich dehnt sich die Blase nach Höhe und Breite, weniger nach der Länge aus".

Bei meinem Tiere fand ich, dass die Hörtasche im Sommer schon in 72 Stunden nach der Befruchtung entsteht und in 84 Stunden zu einer Blase abgeschnürt ist. Das auskleidende Epithel der Blase ist zunächst sehr dünn und nimmt nachher an Höhe zu, aber nicht so schnell, sodass beim Stadium 1 (72 Stunden nach der Befruchtung) (Tafelfig. 1, 2, und Textfig. 1) das Epithel an der dicksten Stelle eine Höhe von $0,01 \mathrm{~mm}$, an der dünnsten eine solche von $0,0026 \mathrm{~mm}$ hat, während bei Stadium 2 (84 Stunden nach der Befruchtung) (Tafelfig. 3, 4, und Textfig. 2) es noch an der dicksten Stelle 0,013 mm, an der dünnsten $0,0026 \mathrm{~mm}$ hoch bleibt. Das Hörbläschen nimmt bald sehr an Breite zu, aber an Höhe und Länge viel weniger, so hat es beim Stadium 1 eine grösste Länge von 0,09 mm, eine Höhe von 0,09-0,1 mm und eine Breite von $0,01 \mathrm{~mm}$, und beim Stadium 2 eine grösste Länge von $0,1 \mathrm{~mm}$, eine Höhe von $0,09 \mathrm{~mm}$ und eine Breite von $0,045 \mathrm{~mm}$.

Im Beginn der Entwickelung hat das Hörbläschen im anteroposterioren und dorsoventralen Durchmesser fast gleiche Masse. Beim Stadium 1 besteht das Epithel aus einschichtig gelagerten Zellen und beim Stadium 2 an der dicksten Stelle aus zwei Zellenlagen, an anderen Stellen aus einschichtigen. Am Stadium 3 (96 Stunden nach Befruchtung) (Tafelfig. 5, 6, und Textfig. 3) erst ist die Anlage der Nervenendstelle angedeutet, wie die Macula recessus utriculi, Macula sacculi und Crista ampullaris posterior, wenngleich sie alle noch miteinander im Zusammenhang stehen; an diesen Stellen besteht das Epithel aus zweischichtig gelagerten Zellen.

Betreffs der Entwickelung der Macula und Crista bei Pelobates sagt Wenig ('13), „dass, wenn sich der Canalis externus bereits abgeschnürt hat, das Neuroepithel folgendermassen geteilt ist: die Crista ampullae externae, die vom utricularen Teile des Neuroepithels ihren Ursprung 
Die Entwickelung des häutigen Labyrinthes des Knochenfisches (Oryzias latipes). 353

nimmt, ist schon vollkommen selbständig, wogegen die Crista ampullae anterioris mit diesem Neuroepithel noch in Verbindung steht. Die Verbindung dieser Crista mit der Macula recessus utriculi hält sehr lange an. Von der Crista ampullae posterioris, Macula sacculi und Papilla lagenae ist die Crista ampullae posterioris zuerst ausgebildet." Diese Teilung des Neuroepithels stimmt mit den Verhältnissen überein, die Norris ('92) bei Amblystoma konstatiert hat.

Bei Oryzias im Stadium 3, wo die beiden vertikalen Bogengänge gebildet sind, ist die Anlage der drei Nervenendstellen, Macula recessus utriculi, Macula sacculi und Crista ampullaris posterior, zu erkennen. Im Stadium 4 (120 Stunden nach der Befruchtung) (Tafelfig. 7, 8, und Textfig. 4), wo sich der laterale Bogengang herausmodelliert ist, trennen sich diese drei Nervenendstellen voneinander. Im Stadium 5 (144 Stunden nach der Befruchtung) (Tafelfig. 9. 10, 11, und Textfig. 5, 6) isoliert sich die Crista ampullaris lateralis erst von der Macula recessus utriculi, obschon hier die Crista ampullaris anterior noch mit der letzteren in Verbindung steht. Die Papilla lagenae isoliert sich am spätesten unter allen Nervenendstellen.

Es ist bei meinem Tiere keine Einteilung in eine Pars inferior und eine Pars superior zu erkennen und auch kein Ductus endolymphaticus kommt zustande.

\section{Die drei Bogengänge und Ampullen.}

Nach Rathke ('61) und Krause ('06) lässt die Bildungsgeschichte der Bogengänge in der Reihe der Wirbeltiere zwei differente Typen erkennen. Zu dem einen gehören die Säugetiere, Sauropsiden und Selachier, bei denen es sich wesentlich um Ausstülpungsvorgänge handelt, während zu dem anderen die Teleostier und Amphibien gehören, welche Einstülpungsprozesse aufweisen, der erstere wird als der Säugertyp, der andere als der Teleostiertyp bezeichnet. Der letzte Autor sagt noch: Bei den Teleostiern treten an die Stelle der Zapfen Septen, indem sich das Epithel der Hörblase einstülpt oder einfaltet und in das Innere der letzteren vordringt. Villy ('90) und Norris ('92) sagen, dass zwei Septen für die beiden vertikalen und ein Septum für den horizontalen Bogengang auftreten. Bei den Teleostiern scheint sich der vordere Bogengang früher zu entwickeln als der hintere und laterale (Noorden ('83)). Nach Okajima ('11) scheint dei Salmo sich der vordere und hintere Bogengang früher zu entwickeln als der laterale.

Bei Oryzias entwickeln sich die beiden vertikalen Bogengänge früher als der laterale, so dass diese schon im Stadium 3 entstehen, 
während der letztere erst im Stadium 4 sich zu bilden kommt. Nach einigen Autoren, Krause ('06) bei allen Wirbeltieren, Fleissig ('08) bei Gecko und Okajima ('12) bei Hynobius, sind die Ampullen schon vor der Ausbildung der Bogengänge angelegt. Nach Norris ('92) erscheinen die Ampullen bei Amblystoma gleichzeitig mit den Bogengängen oder wenigstens sehr bald nach deren Abschnürung. Netto ('98) sagt, dass die Ampullen von Axolotl beim Auftreten der Bogengänge unverkennbar sind. Wenig ('13) schreibt: „Bei den Salmoniden konnte ich jedoch die Ampullen als wirklich unzweifelhafte Gebilde erst längere Zeit nach der Ausbildung der Kanäle sicherstellen ". Dieser Befund stimmt mit dem von Noorden ('83) bei den Teleostiern überein.

Bei meinem Tiere entwickeln sich die Bogengänge früher als die Ampullen. Aber äusserlich bekommen die Ampullen und Bogengänge ihre eigentliche Gestalt um die gleiche Zeit. So treten erst im Stadium 3 die boiden vertikalen Bogengänge auf. Im Stadium 4 deutet sich die Anlage der Ampulla posterior und des lateralen Bogenganges an, und im Stadium 5 kann man die Anlage der Ampulla lateralis finden. Im Stadium 6 (Korperlänge $4 \mathrm{~mm}, 1$ Tag nach Ausbrütung) (Tafelfig. 12, 13, 14 und Textfig. 7, 8) erst erhalten die Ampullen und Bogengänge äusserlich ihre eigentliche Gestalt.

Bezüglich der Winkelbildung zwischen dem vorderen und hinteren Bogengange äussert Krause ('06) die Ansicht, dass die beiden Gänge äusserst stark wachsen und ihr gemeinsamer Schenkel sich nach medial, wo am wenigsten Widerstand ist, vorschiè̀t. Er sagt dabei: „, Dazu kommt noch bei manchen Tieren, z. B. bei den Sauropsiden, ein anderes Moment hinzu. Hier schiebt sich nämlich der sich bildende Sacculus in die Öffnung des horizontalen Bogenganges hinein und drängt so den gemeinsamen Schenkel medialwärts vor." Okajima ('12) schreibt bei Hynobius: „, Auf den Modellen habe ich mit Bestimmtheit gesehen, dass die beiden Ampullen rasch lateralwärts vorrücken, die vordere nach lateral vorn, die hintere nach lateral hinten, wie auch Krause mit Recht sagt, dass die Bildung der Ampullen bei allen Wirbeltieren derjenigen der Bogenänge vorauszueilen scheint. Ich möchte daraus schliessen, dass die Winkelbildung der Vorschiebung der Bogengänge durch die wachsenden Ampullen zu verdanken ist."

Bei Oryzias habe ich auf den Modellen (Stadium 10, ausgewachsenes Exemplar) gesehen, dass die beiden Ampullen nicht nur rasch lateralwärts vorrücken, die vordere nach lateral vorn, die hintere nach lateral hinten, wio es Okajima bei Hynobius sah, sondern sie auch noch 
Die Entwickelung des häutigen Labyrinthes des Knochenfisches (Oryzias latipes). 355 aussen unten umdrehen und abwärts herabsteigen. Daraus möchte ich schliessen, dass die Winkelbildung der beiden vertikalen Bogengänge hauptsïchlich auch durch die Verlagerung der beiden wachsenden Ampullen hervorgerufen wird.

Die mediale und laterale Wand der Bogengünge sind zuerst seitlich abgeplattet, werden aber allmählich vorgetrieben, bis endlich der vordere Bogengang auf Querschnitt elliptisch, indem seine obere und untere Wand flach bleiben, und der laterale und hintere zylindrisch wird.

Die Ampulla posterior liegt zuerst im Stadium 6 höher als andere zwei Ampullen, welche zusammen weiter unten in gleicher Höhe stehen. $\Lambda$ ber beim Fortschreiten der Stadien steigen die vordere und hintere Ampulle immer mehr nach unten herab und endlich kommt beim ausgewachsenen Exemplar die Ampulla lateralis höher als die anderen zwei zu stehen. Diese Höhenver:̈̈nderung der Ampullen wird durch die schon óben angegebene Drehung und Verlängerung der beiden vertikalen hervorgerufell. Die Gestalt der Ampulla anterior und posterior beim ausgewachsenen Tiere ist rundlich und die der Ampulla lateralis, der grössten von den drei, tonnenförmig.

Die Septumbildung für den vorderen und hinteren Bogengang entsteht bei Oryzias erst im Stadium 3, die für den lateralen im Stadium 4. So treten je zwei mediale und laterale Septen für die beiden vertikalen und ein unteres Septum für den lateralen Bogengang auf, wie Villy ('90) und Norris ('92) bei Frosch und Amblystoma fanden. Das Septum nervum tritt erst am Stadium 8 (Körperlänge $9 \mathrm{~mm}, 15$ Tage nach Ausbrütung) auf.

\section{Andere Teile des Labyrinthes.}

Alle Teile des Labyrinthes von Fischen sind schon von Retzịus ('81) eingehend beschrieben worden, aber deren Entwickelungsgeschichte ist wenig bekannt. Krause ('06) schreibt bei Forellenembryo: "Schon zu der Zeit, wenn eben die ersten Anzeichen der Bogengangsbildung einsetzen, treibt das Hörbläschen ventralwärts eine Ausstülpung von ungefähr rhombischer Gestalt. Zunächst zeigt sie das Bestreben, sich kaudalwärts auszudehnen, so dass ihre Spitze dann, wenn die Bogengänge völlig durchgebrochen sind, die hintere Ampulle noch überragt. Beim weiteren Wachstum aber dehnt sie sich auch zerebralwärts aus und schiebt sich unter dem Boden des Utriculus nach vorn. Dadurch setzt sich der Sacculus immer mehr gegen den Utriculus samt den Bogengängen ab. Noch aber kommunizieren beide durch eine breite Öffnung miteinander." 
Bei ineinem Tiere stellt das Labyrinth, wenngleich die Bogengänge. schon völlig durchgebrochen sind (Stadium 3-4), noch einen einheitlichen Raum dar. Vom Stadium 6 an sieht man zum ersten Male die Differenzierung seiner einzelnen Teile, wie des Utriculosacculus, Sinus utriculi superior, Sinus utriculi posterior und der Lagena cochleae, welche alle immer deutlicher sich gestalten. Erst am Stadium 9 (Körperlänge $13 \mathrm{~mm}$, 35 Tage nach Ausbrütung) (Tafelfig. 21, 22, 23 und Textfig. 11) ist der Recessus utriculi durch eine deutliche Einschnürung von dem Utriculosacculus abgesetzt.

Der Utriculosacculus. Bei Oryzias zeigt mein sogenannter Utriculosacculus eine besondere Gestalt als bei den übrigen Teleostiern, so dass er eine ganz einheitliche Höhle aufweist und keine Andeutung der Teilung in einen Utriculus und einen Sacculus darbietet. Erst von Stadium 5 an ist er von anderen 'Teilen abgesetzt und trägt eine mit der Längsachse anteroposterior stehende Tonnenform. Der vordere Teil seiner oberen Wand neigt sich nach vorn unten, der hintere Teil nach hinten unten sehr stark. Vorn geht er in den Recessus utriculi, hinten in den Sinus utriculi posterior und die Lagena cochleae und oben in den Sinus utriculi superior über. Bei weiterer Entwickelung dehnt sich seine laterale Wand lateralwärts aus, waihrend die mediale abgeplattet ist. Im Stadium 9 neigt sich der vordere Teil seiner lateralen Wand schief nach innen vorn, und zwischen dem Utriculosacculus und Recessus utriculi liegt eine deutliche Einschnürung. Beim ausgewachsenen Exemplar (Tafelfig. 24, 25, 26 und Textfig. 12, 13, 14) weicht der untere Teil der lateralen Wand stark nach innen unten ab und bildet mit der platten medialen Wand sich verbindend eine scharfe untere Kante, während deren vorderer Teil stark nach innen vorn geneigt ist; er kommuniziert durch einen seitlich engen platten Kanal mit dem Recessus utriculi. Der obere Teil seiner hinteren Wand zeigt sich als eine senkrechte Ebene und geht oben in die hintere Wand des Sinus utriculi superior über, während der untere Teil nach hinten etwas angeschwollen ist und nach aussen hinten in den Sinus utriculi posterior, nach innen hinten in die Lagena cochleae übergeht.

Der Recessus utriculi. Im Stadium 5 ist er vom Utriculosacculus durch eine schwache Einschnürung abgesetzt, die sich bei weiterem Wachstum allmählich verticft. Beim ausgewachsenen ragt er vom Utriculosacculus schief nach vorn aussen hervor und hat eine platte äussere obere Wand und einen kugligen Boden.

Der Sinus utriculi superior. Im Stadium 5 ist er noch sehr kurz und klein, vergrössert sich aber von Zeit zu Zeit, so dass er beim 
Die Entwickelung des häutigen Labyrintbes des Knochenfisches (Oryzias latipes). 357 ausgewachsenen breit wird und sein oberer Teil nach aussen innen etwas angeschwollen ist ; die obere Wand ist abgeplattet und trägt keinen Apex.

Der Sinus utriculi posterior. Am Stadium 5 ist dieser Teil kaum erkennbar und geht von dem Utriculosacculus nach hinten aussen aus; seine mediale und laterale Wand sind flach. Bei weiterem Wachstum wird er allmählich zylindrisch und länger. Beim ausgewachsenen ist er zylindrisch und ragt vom hinteren äusseren unteren Ende des Utriculosacculus nach aussen stark geknickt hervor. Er stellt bei Oryzias einen vom Utriculosacculus getrennten, mehr oder weniger selbständigen Raum dar und hat keinen direkten Zusammenhang mehr mit dem wesentlichen Utriculusteil, während er bei den anderen Teleostiern die hintere Fortsetzung des Utriculus bildet.

Die Lagena cochleae. Bei meinem Tiere ragt die Lagena erst im Stadium 6 links sehr schwach vom hinteren inneren unteren Ende des Utriculosacculus nach hinten innen hervor, während rechts noch keine Andeutung davon zu sehen ist, was erst im Stadium 7 stattfindet. Bei weiterem Wachstum springt die Lagena nach hinten innen hervor. Beim ausgewachsenen ist sie rundlich und kommuniziert durch eine grosse rundliche Öffnung mit dem Utriculosacculus. Sie scheint bei Oryzias unter allen Labyrinthteilen sich am spätesten zu entwickeln.

\section{Ergebnisse.}

Die Resultate der vorliegenden Untersuchung lassen sich folgendermassen zusammenfassen.

Das häutige Labyrinth des Oryzias weicht in einigen Punkten von dem Teleostiertypus ab.

1. Das Hörbläschen entsteht viel später als das Augenbläschen und früher als das Riechfeld. Im Beginn der Entwickelung sind sein anteroposteriorer Durchmesser und der dorsoventrale lang und der mediolaterale sehr kurz; so stellt es eine seitlich abgeplattete Blase dar. Nach und nach aber erweitert es sich mediolateral sehr, dann anteroposterior und dorsoventral. Vom Anfang an ist eine Begrenzung zwischen der Pars inferior und Pars superior nicht zu erkennen.

2. Die beiden vertikalen Bogengänge scheinen sich früher zu entwickeln als der laterale. Und die Bogengänge scheinen sich auch früher zu entwickeln als die Ampullen, wenngleich es sich um fast die gleiche Zeit handelt, dass die Ampullen und Bogengänge äusserlich ihre eigentliche Gestaltung vervollständigen. Zuerst werden die drei Nervenendstellen, die Macula sacculi, Macula recessus utriculi und Crista ampul- 
laris posterior angelegt und dann die Crista ampullaris lateralis und anterior. Die Papilla lagenae entwickelt sich am spätesten von allen Nervenendstellen.

3. Die Winkelbildung zwischen dem vorderen und hinteren Bogengange kommt dadurch zustande, dass diese beiden weiter an Länge wachsen und der vordere nach lateral vorn, der hintere nach lateral hinten sich ausdehnen und die beiden Ampullen nach lateral unten herabsteigend sich umdrehen.

4. Der Hochstand der lateralen Ampulle wird durch die laterale untere Umdrehung und Absteigung der vorderen und hinteren Ampullen, welche anfangs viel höher als jene standen, hervorgerufen.

5. Der Utriculus und der Sacculus weisen zusammen einen ganz einheitlichen Hohlraum auf, den ich den Utriculosacculus nenne, und es ist keine Grenze zwischen den beiden vorhanden im Gegensatz zu den anderen Teleostiern, wo eine mehr oder weniger deutliche Grenze vorhanden ist; daher ist hier kein Foramen (Canalis) utriculosacculare zu finden. Der Recessus utriculi kommuniziert durch eine seitlich verengerte Öffnung mit dem Utriculosacculus. Der Sinus utriculi posterior stellt einen vom Utriculosacculus getrennten, mehr oder weniger selbständigen Raum dar und hat keinen direkten Zusammenhang mehr mit dem wesentlichen Utriculusteil, während er bei den anderen Teleostiern die hintere Fortsetzung des Utriculus bildet.

Die Macula neglecta und der Ductus endolymphaticus fehlen beide beim Oryzias vollständig.

Zum Schluss möchte ich Herrn Prof. Dr. K. Okajima für seine dauernde Unterstützung bei dieser Arbeit meinen herzlichsten Dank aussprechen.

\section{Literatur.}

1. Alexander, C., Über das Gehörorgan der Ganoiden. Arch. f. mikr. Anat. Bd. 18. 1880.

2. Fleissig, J., Die Entwickelung des Geckolabyrinthes. Anat. Hefte. Bd. 37. 1908.

3. Hasse, Das Gehörorgan der Fische. Anat. Studien. Heft. III. I872.

4. Hertwig, O., Handbuch d. vergl. u. experim. Entwickelungslehre. Bd. 2. II. Teil. 1906.

5. Keibel, Über die Entwickelung des Labyrinthanhangs. Anat. Anz. Bd. 16. 1899.

6. Krause, R, Entwickelungsgeschichte d. häutigen Bogenganges. Arch. f. mikr. Anat. Bd. 35. 1890. 
Die Entwickelung des häutigen Labyrinthes des Knochenfisches (Oryzias latipes). 359

7. - - Entwickelungsgeschichte des Gehörorgans. Hertwigs Handbuch. 1906.

8. Kuhn, Untersuchungen über das häutige Labyrinth der Knochenfische. Arch. f. mikr. Anat. Bd. 16. 1877.

9. Netto, F., Die Entwickelung des Gehörorgans beim Axolotl. Inaug. Diss. Berlin 1898 (Ref. aus Wenig).

10. Noorden, C. V., Die Entwickelung des Labyrinthes bei Knochenfischen. Arch. f. Anat. u. Phys. Anat. Abt. 1883.

11. Norris, H.W., Studien über die Entwickelung des Ohres bei Amblystoma. Journ. of Morph. Vol. 7. 1892. (Ref. bei Wenig).

12. Okajima, K., Die Entwickelung des Gehörorgans von Hynobius. Anat. Hefte. Bd. 45. 1912.

13. — - Die Entwickelung der Macula neglecta beim Salmoembryo. Anat. Anz. Bd. 40. 1911.

14. Poli, C., Zur Entwickelung der Gehörblase bei den Wirbeltieren. Arch. f. mikr. Anat. Bd. 48. 1897.

15. Retzius, G., Zur Kenntnis von dem membranösen Gehörlabyrinth bei den Knorpelfischen. Arch. f. Anat. u. Phys. Anat. Abt. II. u. III. Hefte. 1878.

16. —, Das Gehörorgan der Wirbeltiere. 1. Das Gehörorgan der Fische und Amphibieu. Stockholm 1881.

17. Sidoriak, Zur Entwickelung des endolymphatischen Apparats bei den Fischen. Anat. Anz. Bd. 15. 1898.

18. Thompson W., On the auditory Labyrinth of Orthagoriscus mola L. Anat. Anz. Bd. III. 1888.

19. Villy, F. The development of the ear and the accessory organs in the common Frog. Quart. Journ. micr. Sc. Vol. XXX. 1890. (Ref. bei Wenig).

20. Vogt, C., Embryologie des Salmones. Neuchatel 1842 (Ref. bei Noorden).

21. Wenig, J., Untersuchungen über die Entwickelung der Gehörorgane der Anamnia. Morph. Jahrb. Bd. 45. 1913.

22. — - Die Entwickelung des Ductus endolymphaticus bei den Knochenfischen. Anat. Anz. Bd. 33. 1911.

\section{Figurenerklärung.}

Wachsplattenmodelle des häutigen Labyrinthes.

Alle Figuren sind in natürlicher Grösse der Modelle gezeichnet.

Fig. 1. Stadium 1. Laterale Ansicht (links, $100 \mathrm{fach}$ ).

Fig. 2. Stadium 1. Mediale Ansicht ( ,, , ).

Fig. 3. Stadium 2. Laterale Ansicht (rechts, 100 fach).

Fig. 4. , Mediale ,, (,, ,, ).

Fig. 5. Stadium 3. Laterale Ansicht (rechts, $100 \mathrm{fach}$ ).

Fig. 6. ", Mediale ,, ( , ," ).

Fig. 7. Stadium 4. Laterale Ansicht (rechts, 100 fach).

Fig. 8. ," Mediale ," (, , ). 
Fig. 9. Stadium 5. Laterale Ansicht (rechts, 100 fach).

Fig. 10. ," Mediale , ( , , ).

Fig. 11. " Obere ", ( , ,").

Fig. 12. Stadium 6. Laterale Ansicht (rechts, 100 fach).

Fig. 13. ", Mediale ", ( , , ).

Fig. 14. ", Obere ", ( , , ).

Fig. 15. Stadium 7. Laterale Ansicht (rechts, 66 fach).

Fig. 16. , Mediale ,, ( , , ).

Fig. 17. ", Obere ", ( , ,").

Fig. 18. Stadium 8. Laterale Ansicht (rechts, $50 \mathrm{fach}$ ).

Fig. 19. , Mediale ," ( , , ).

Fig. 20. ", Obere ", ( , , ).

Fig. 21. Stadium 9. Laterale Ansicht (links, $50 \mathrm{fach}$ ).

Fig. 22. ", Mediale ," ( , ", ).

Fig. 23. ", Obere ", ( , ", ).

Fig. 24. Stadium 10. Laterale Ansicht (links, 33 fach).

Fig. 25. , Mediale ,, ( , , ).

Fig. 26. ", Obere ", ( , , ).

\section{Abkrüzungen.}

A.v.B. Anlage des vorderen Bogenganges.

A.h.B. Anlage des hinteren Bogenganges.

A.l.B. Anlage des lateralen Bogenganges.

v.B. Vorderer Bogengang.

1.B. Lateraler Bogengang.

h.B. Hinterer Bogengang.

A.a. Ampulla anterior.

A.l. Ampulla lateralis.

A.p. Ampulla posterior.

$\mathrm{S}^{1} \quad$ Laterales Septum für den vorderen Bogengang.

$\mathrm{S}^{2} \quad$ Laterales Septum für den hinteren Bogengang.

$\mathrm{S}^{3} \quad$ Mediales Septum für den vorderen Bogengang.

$S^{4} \quad$ Mediales Septum für den hinteren Bogengang.

$\mathrm{S}^{5} \quad$ Oberes Septum für den lateralen Bogengang.

$\mathrm{S}^{6} \quad$ Unteres Septum für den lateralen Bogengang.

R.u. Recessus utriculi.

S.s. Sinus utriculi superior.

S.p. Sinus utriculi posterior.

U-s. Utriculosacculus.

L. Lagena.

Cr.a. Crista ampullaris anterior.

Cr.1. Crista ampullaris lateralis.

Cr.p. Crista ampullaris posterior.

M.u. Macula utriculi.

M.s. Macula sacculi.

P.1. Papilla lagenae. 
FOLIA ANATOMICA JAPONICA, BD. V.

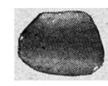

Fig. 1.

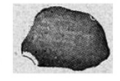

Fig. 2.

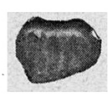

Fig. 3.

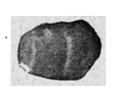

Fig. 4 .
TAF. XXXVIII.

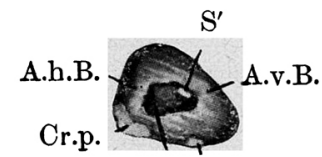

$\mathrm{S}^{2}$ M.u.

Fig. 5.

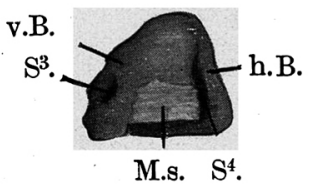

Fig. 8.

Fig. 6 .

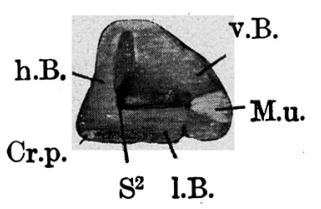

Fig. 7.

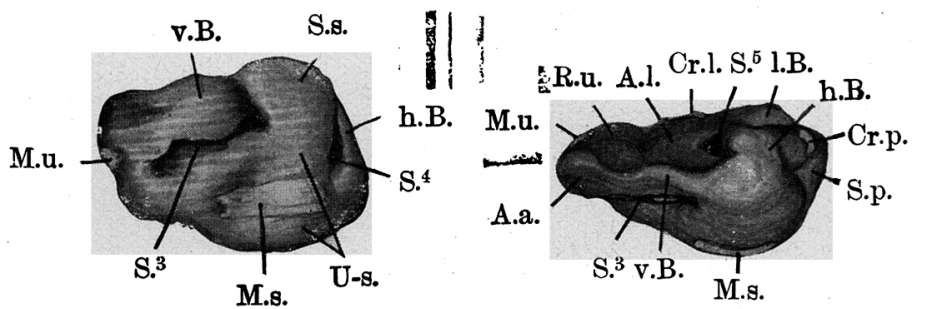

Fig. 11.

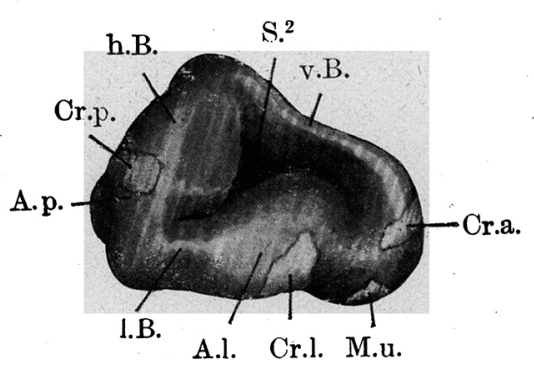

Fig. 1?.

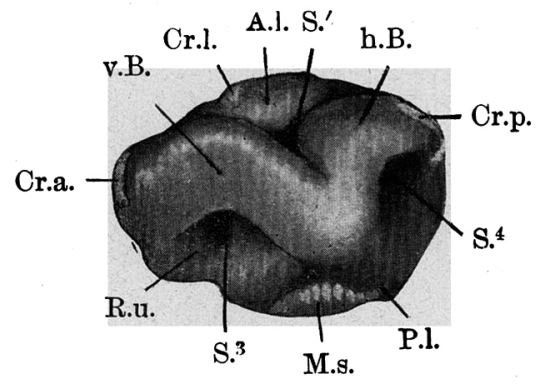

Fig. 14.

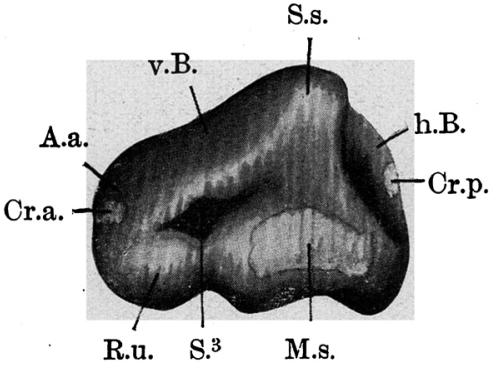

Fig. 13.

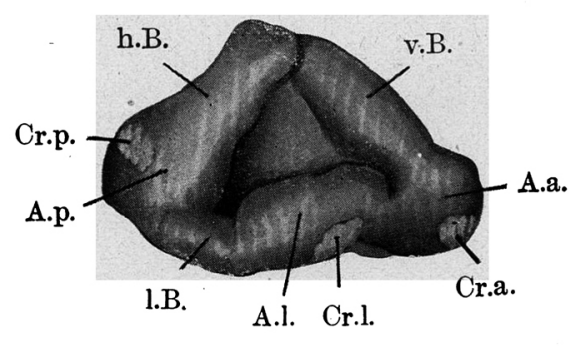

Fig. 15.

T. Murayama. 
FOLIA ANATOMICA JAPONICA, BD. V.

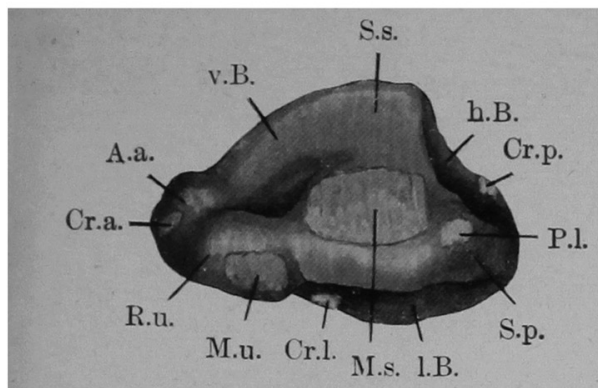

Fig. 16.

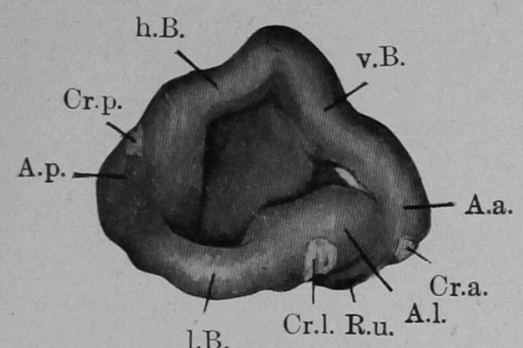

Fig. 18.

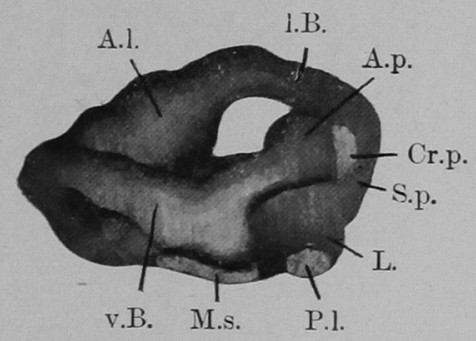

Fig. 20.

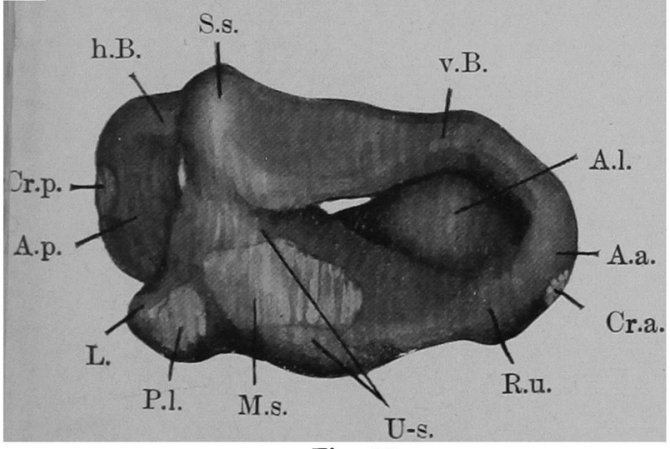

Fig. 22 .
TAF. XXXIX.

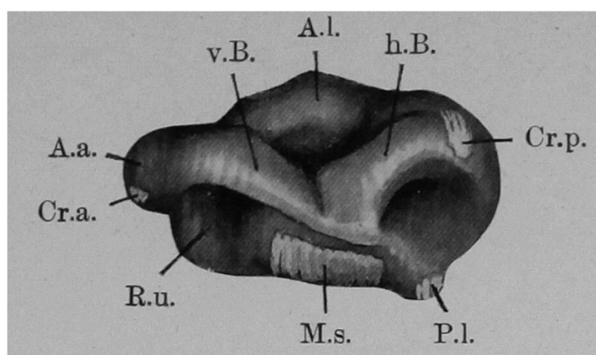

Fig. 17.

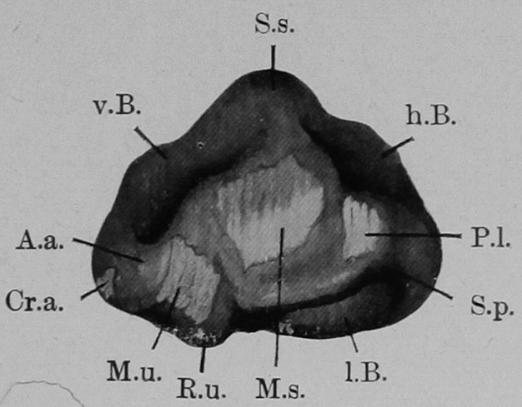

Fig. 19

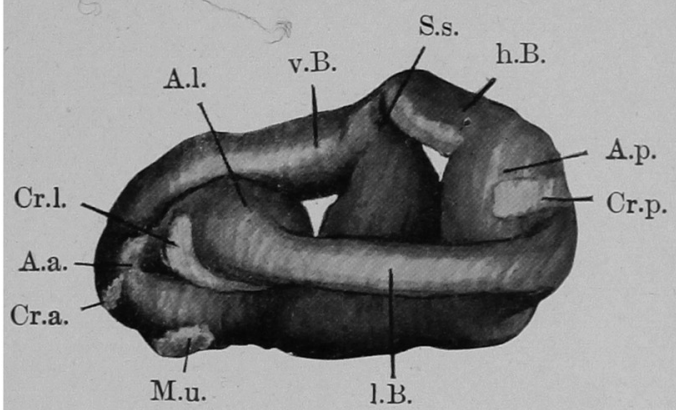

Fig. 21.

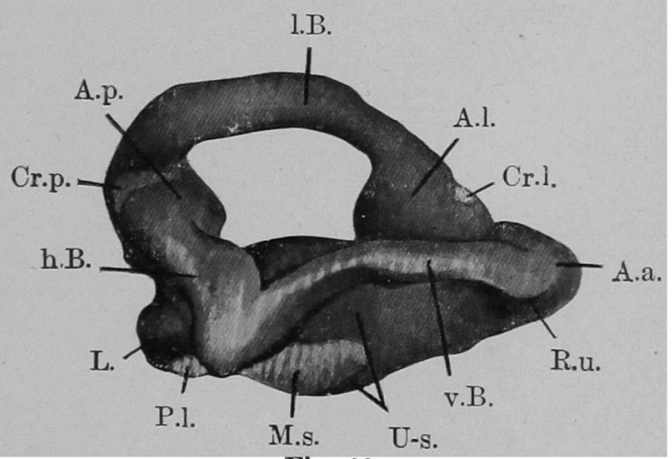

Fig. 23.

T. Murayama 
FOLIA ANATOMICA JAPONICA, BD. V.

TAF. XL

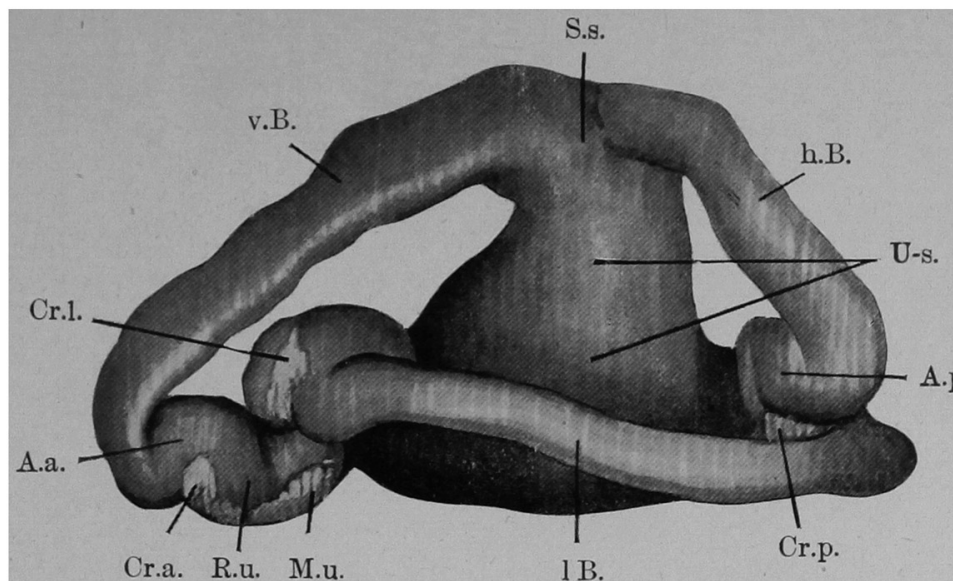

Fig. 24.

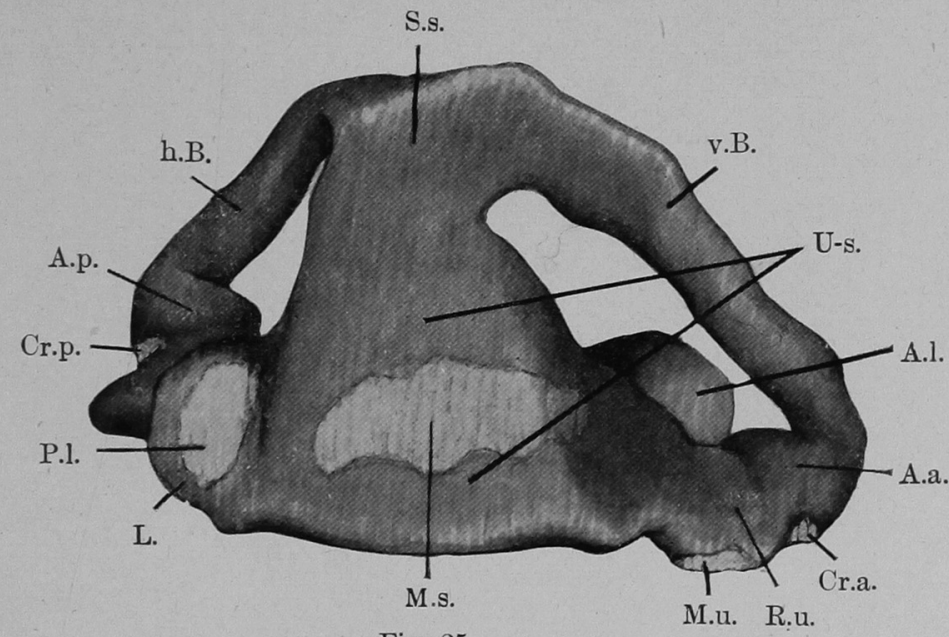

Fig. 25 .

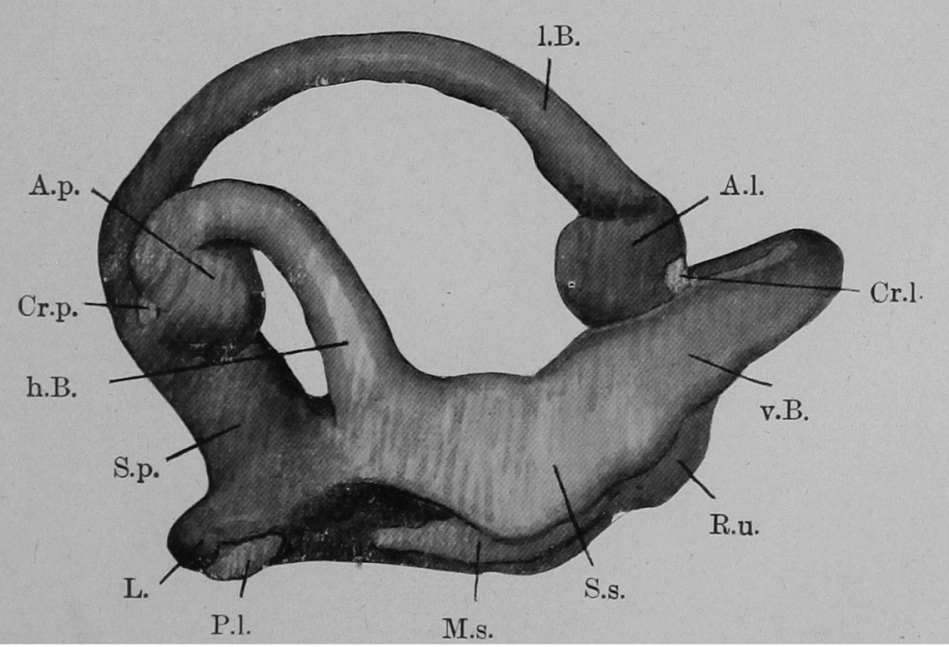

T. Murayama.

Fig. 26. 